\title{
KILLING VECTOR FIELDS OF CONSTANT LENGTH ON COMPACT HOMOGENEOUS RIEMANNIAN MANIFOLDS
}

\author{
YU. G. NIKONOROV
}

\begin{abstract}
In this paper we present some structural results on the Lie algebras of transitive isometry groups of a general compact homogenous Riemannian manifold with nontrivial Killing vector fields of constant length.

2000 Mathematical Subject Classification: 53C30 (primary), 53C20, 53C25, 53C35 (secondary).

Key words and phrases: Clifford-Wolf homogeneous spaces, geodesic orbit spaces, homogeneous spaces, Hermitian symmetric spaces, homogeneous Riemannian manifolds, Killing vector fields of constant length.
\end{abstract}

\section{INTRODUCTION AND THE MAIN RESULTS}

Recall that a vector field $X$ on a Riemannian manifold $(M, g)$ is called Killing if $L_{X} g=0$. In this paper, we study Killing vector fields of constant length on homogeneous Riemannian manifolds. A detailed study of Killing vector fields of constant length was started in the papers $[5,6,7]$, although many of the results in this direction have long been known (see a detailed exposition in [5]). It should be noted that there exists a connection between Killing vector fields of constant length and Clifford-Wolf translations in a Riemannian manifold $(M, g)$.

Recall that a Clifford-Wolf translation in $(M, g)$ is an isometry $s$ moving all points in $M$ one and the same distance, i. e. $\rho_{g}(x, s(x)) \equiv$ const for all $x \in M$, where $\rho_{g}$ means the inner (length) metric generated by the Riemannian metric tensor $g$ on $M$. CliffordWolf translations naturally appear in the investigation of homogeneous Riemannian coverings of homogeneous Riemannian manifolds [18, 32]. Clifford-Wolf translations are studied in various papers (see e.g. [6, 16, 29, 30, 25] for the Riemannian case and $[13,14,15]$ for the Finsler case), for a detailed discussion we refer to [8] and [34].

If a one-parameter isometry group $\gamma(t)$ on $(M, g)$, generated by a Killing vector field $Z$, consists of Clifford-Wolf translations, then $Z$ obviously has constant length. This assertion can be partially inverted: If a Riemannian manifold $(M, g)$ has the injectivity radius, bounded from below by some positive constant (in particularly, this condition is satisfied for every compact or homogeneous manifold), and $Z$ is a Killing vector field of constant length on $(M, g)$, then the isometries $\gamma(t)$ from the 1-parameter isometry group, generated by the vector field $Z$, are Clifford-Wolf translations at least for sufficiently small $|t|[6]$.

A metric space $(M, \rho)$ is Clifford-Wolf homogeneous if for any points $x, y \in M$ there exists an isometry $f$, Clifford-Wolf translation, of the space $(M, \rho)$ onto itself such that $f(x)=y$. A connected Riemannian manifold $(M, g)$ is Clifford-Wolf homogeneous if it is Clifford-Wolf homogeneous relative to its inner metric $\rho_{g}$. In addition, it is $G$ Clifford-Wolf homogeneous if one can take isometries $f$ from the Lie (sub)group $G$ of isometries of $(M, g)$ in the above definition of Clifford-Wolf homogeneity. Clifford-Wolf homogeneous simply connected Riemannian manifold are classified in [8]: A simply 
connected Riemannian manifold is Clifford-Wolf homogeneous if and only if it is a direct metric product of an Euclidean space, odd-dimensional spheres of constant curvature and simply connected compact simple Lie groups supplied with bi-invariant Riemannian metrics. Note that every geodesic $\gamma$ in a Clifford-Wolf homogeneous Riemannian manifold $(M, g)$ is an integral curve of a Killing vector field of constant length on $(M, g)[8]$.

In a recent paper [34], Ming Xu and Joseph A. Wolf obtained the classification of normal Riemannian homogeneous spaces $G / H$ with nontrivial Killing vector fields of constant length, where $G$ is compact and simple. Every of these spaces with $\operatorname{dim}(G)>$ $\operatorname{dim}(H)>0$ is locally symmetric and its universal Riemannian cover is either an odd-dimensional sphere of constant curvature, or a Riemannian symmetric space $S U(2 n) / S p(n)$. This result is very important in the context of the study of general Riemannian homogeneous manifolds with nonzero Killing fields of constant length. In a very recent paper [33], this result was extended to the class of pseudo-Riemannian normal homogeneous spaces.

In this paper we present some structural results on the Lie algebras of transitive isometry groups of a general compact homogenous Riemannian manifold with nontrivial Killing vector fields of constant length. All manifolds supposed connected throughout this paper.

Let us consider any Lie group $G$ acting on the Riemannian manifold $(M, g)$ by isometries. The action of $a$ on $x \in M$ will be denoted by $a(x)$. We will identify the Lie algebra $\mathfrak{g}$ of $G$ with the corresponded Lie algebra of Killing vector field on $(M, g)$ as follows. For any $U \in \mathfrak{g}$ we consider a one-parameter group $\exp (t U) \subset G$ of isometries of $(M, g)$ and define a Killing vector field $\widetilde{U}$ by a usual formula

$$
\widetilde{U}(x)=\left.\frac{d}{d t} \exp (t U)(x)\right|_{t=0} .
$$

It is clear that the map $U \rightarrow \widetilde{U}$ is linear and injective, but $[\widetilde{U}, \widetilde{V}]=-\widetilde{[U, V]}$. We will use this identification repeatedly in this paper.

Let $(M, g)$ be a compact connected Riemannian manifold, $G$ is a transitive isometry group of $(M, g)$. We identify elements of the Lie algebra $\mathfrak{g}$ of $G$ with Killing vector fields on $(M, g)$ as above. Since $G$ is compact, then we have a decomposition

$$
\mathfrak{g}=\mathfrak{c} \oplus \mathfrak{g}_{1} \oplus \mathfrak{g}_{2} \oplus \cdots \oplus \mathfrak{g}_{k},
$$

where $\mathfrak{c}$ is the center and $\mathfrak{g}_{i}, i=1, \ldots, k$, are simple ideals in $\mathfrak{g}$.

We are going to state the main results of this paper, that are formulated under the above assumptions and notations.

Theorem 1. Let $Z=Z_{0}+Z_{1}+Z_{2}+\cdots+Z_{l} \in \mathfrak{g}$ be a Killing vector field of constant length on $(M, g)$, where $1 \leq l \leq k, Z_{0} \in \mathfrak{c}, Z_{i} \in \mathfrak{g}_{i}$ and $Z_{i} \neq 0$ for $1 \leq i \leq l$. Then the following statements hold:

1) For every $i \neq j, 1 \leq i, j \leq l$, we have $g\left(\mathfrak{g}_{i}, \mathfrak{g}_{j}\right)=0$ at every point of $M$. In particular, $g\left(Z_{i}, \mathfrak{g}_{j}\right)=0$ and $g\left(Z_{i}, Z_{j}\right)=0$.

2) Every Killing field of the type $Z_{0}+Z_{i}, 1 \leq i \leq l$, has constant length.

Conversely, if for every $i, 1 \leq i \leq l$, the Killing field $Z_{0}+Z_{i}$ has constant length, and for every $i \neq j, 1 \leq i, j \leq l$, the equality $g\left(\mathfrak{g}_{i}, \mathfrak{g}_{j}\right)=0$ holds, then the Killing field $Z=Z_{0}+Z_{1}+Z_{2}+\cdots+Z_{l}$ has constant length on $(M, g)$. 
Corollary 1. If (under the assumptions of theorem 1) we have $\mathfrak{c}=0$ and $k=l$, then every $\mathfrak{g}_{i}, 1 \leq i \leq k$, is a parallel distribution on $(M, g)$. Moreover, if $(M, g)$ is simply connected, then it is a direct metric product of $k$ Riemannian manifolds.

Theorem 1 allows to restrict our attention on Killing vector fields of constant length of the following special type: $Z=Z_{0}+Z_{i}$, where $Z_{0}$ is in the center $\mathfrak{c}$ of $\mathfrak{g}$ and $Z_{i}$ is in the simple ideal $\mathfrak{g}_{i}$ in $\mathfrak{g}$. Without loss of generality we will assume that $i=1$.

Theorem 2. Let $Z=Z_{0}+Z_{1} \in \mathfrak{g}$ be a Killing vector fields of constant length on $(M, g)$, where $Z_{0} \in \mathfrak{c}, Z_{1} \in \mathfrak{g}_{1}$ and $Z_{1} \neq 0$, and let $\mathfrak{k}$ be the centralizer of $Z$ (and $Z_{1}$ ) in $\mathfrak{g}_{1}$. Then either any $X \in \mathfrak{g}_{1}$ is a Killing field of constant length on $(M, g)$, or the pair $\left(\mathfrak{g}_{1}, \mathfrak{k}\right)$ is one of the following irreducible Hermitian symmetric pair:

1) $(s u(p+q), s u(p) \oplus s u(q) \oplus \mathbb{R}), p \geq q \geq 1$;

2) $(s o(2 n), s u(n) \oplus \mathbb{R}), n \geq 5$;

3) $(s o(p+2), s o(p) \oplus \mathbb{R}), p \geq 5$;

4) $(s p(n), s u(n) \oplus \mathbb{R}), n \geq 2$;

In the latter four cases the center of $\mathfrak{k}$ is a one-dimensional Lie algebra spanned by the vector $Z_{1}$.

If a Killing field of constant length $Z=Z_{0}+Z_{1}$ satisfies one of the cases 1)-4) in theorem 2, we will say that it has Hermitian type. Recall that an element $U \in \mathfrak{g}$ is regular in $\mathfrak{g}$, if its centralizer has minimal dimension among all the elements of $\mathfrak{g}$. For $Z$ of Hermitian type, $Z_{1}$ is not a regular element in $\mathfrak{g}_{1}$, since $\mathfrak{k}$ is not commutative in the cases 1)-4) of theorem 2. Hence, we get

Corollary 2. If $Z_{1}$ is a regular element in the Lie algebra $\mathfrak{g}_{1}$ (under the assumptions of theorem 2), then every $X \in \mathfrak{g}_{1}$ is a Killing vector field of constant length on $(M, g)$.

Moreover, the following result holds.

Theorem 3. If $Z=Z_{0}+Z_{1}+\cdots+Z_{k}$ is a regular element of $\mathfrak{g}$ and has constant length on $(M, g)$, then the following assertions hold:

1) $g\left(\mathfrak{g}_{i}, \mathfrak{g}_{j}\right)=0$ for every $i \neq j, i, j=1, \ldots, k$, at every point of $M$;

2) $g\left(Z_{0}, \mathfrak{g}_{i}\right)=0$ for every $i=1, \ldots, k$, at every point of $M$;

3) every $X \in \mathfrak{g}_{\mathfrak{s}}$, where $\mathfrak{g}_{\mathfrak{s}}=\mathfrak{g}_{1} \oplus \cdots \oplus \mathfrak{g}_{k}$ is a semisimple part of $\mathfrak{g}$, has constant length on $(M, g)$.

Moreover, if $M$ is simply connected, then $Z_{0}=0$ and $(M, g)$ is a direct metric product of $\left(G_{i}, \mu_{i}\right), 1 \leq i \leq k$, where $G_{i}$ is a connected and simply connected compact simple Lie group with the Lie algebra $\mathfrak{g}_{i}$ and $\mu_{i}$ is a bi-invariant Riemannian metric on $G_{i}$.

The structure of the paper is the following. We consider various examples of Killing vector fields of constant length in section 1 . Sections 2,3 , and 4 are devoted to the presentation of auxiliary results on Killing vector fields on Riemannian manifolds, on some algebraic properties of special homogeneous spaces, and on the root spaces decompositions of simple Lie algebras. In section 5 we prove theorems 1 and 3, corollary 1 and start to prove theorem 2. We finish the proof of theorem 2 in section 6 . Section 7 devoted to a more detailed study of cases 1)-4) in theorem 2. Finally, we discuss some unsolved problems and questions in section 8.

Acknowlegment. The author extends gratitude to Professor V.N. Berestovskii for interesting and helpful discussions concerning this project and to the anonymous 
referee for helpful comments and suggestions that improved the presentation of this paper.

\section{Examples of Killing VECTOR Fields of CONSTANT LENGTH}

In this section we discuss some examples of Killing vector fields of constant length on Riemannian manifolds. At first we consider examples for theorem 2 with simple $\mathfrak{g}_{1}$ consisting of Killing fields of constant length.

It is well known that the group of left translations, as well as the group of right translation, of a compact Lie group $G$, supplied with a bi-invariant Riemannian metric $\mu$, consists of Clifford-Wolf translations. Therefore, Killing fields that generate these groups have constant length on $(G, \mu)$. Of course, every direct metric product of $(G, \mu)$ with any Riemannian manifolds also has Killing fields of constant length. Now, let us consider more interesting examples.

Let $F$ be a connected compact simple Lie group, $k \in \mathbb{N}$. Consider a so-called Ledger - Obata space $F^{k} / \operatorname{diag}(F)$, see [21, section 4] or [22]. We supply it with any invariant Riemannian metric $g$. The structure of invariant Riemannian metrics on $F^{k} / \operatorname{diag}(F)$ is discussed in details in [22]. It should be noted that for any compact Lie group $F$, a Ledger - Obata space $F^{k} / \operatorname{diag}(F)$ is diffeomorphic to the Lie group $F^{k-1}\left[21\right.$, P. 453]. It is easy to see that every copy $F$ in $F^{k}$ consists of CliffordWolf translations on $\left(F^{k} / \operatorname{diag}(F), g\right)$ as well as every copy of the Lie algebra $\mathfrak{f}$ in $\mathfrak{f} \oplus \mathfrak{f} \oplus \cdots \oplus \mathfrak{f}=k \cdot \mathfrak{f}$ consists of Killing fields of constant length for any invariant Riemannian metric $g$. For example, we may consider $g$ induced with the Killing form of $k \cdot \mathfrak{f}$. For such a choice $\left(F^{k} / \operatorname{diag}(F), g\right)$ is (locally) indecomposable.

The above example could be generalized as follows. Consider a compact simple Lie group $F$ and compact Lie groups $G_{1}, G_{2}, \ldots, G_{l}$ such that every $G_{i}$ has a subgroup isomorphic to $F \times K_{i}$. Then the group $F \times G_{1} \times \cdots \times G_{l}$ acts naturally on $M=$ $G_{1} / K_{1} \times \cdots \times G_{l} / K_{l}$ :

$$
\left(a, b_{1}, b_{2}, \ldots, b_{l}\right) \cdot\left(c_{1} K_{1}, c_{2} K_{2}, \ldots, c_{l} K_{l}\right) \rightarrow\left(b_{1} c_{1} K_{1} a^{-1}, b_{2} c_{2} K_{2} a^{-1}, \ldots, b_{l} c_{l} K_{l} a^{-1}\right) .
$$

Hence, $M=\left(F \times G_{1} \times \cdots \times G_{l}\right) /\left(K_{1} \times \cdots \times K_{l} \times \operatorname{diag}(F)\right)$. It is clear that for any $\left(F \times G_{1} \times \cdots \times G_{l}\right)$-invariant Riemannian metric $g, F$ consists of Clifford-Wolf translation on $(M, g)$ and every $Z \in \mathfrak{f}$ has constant length on $(M, g)$, because the group $G_{1} \times \cdots \times G_{l}$ is transitive on $(M, g)$.

In what follows, we will consider examples for the cases 1)-4) in theorem 2.

We say that a Lie algebra $\mathfrak{g}$ of Killing vector fields is transitive on a Riemannian manifold $(M, g)$ if $\mathfrak{g}$ generates the tangent space to $M$ at every point $x \in M$, or, equivalently, the connected isometry group $G$ with the Lie algebra $\mathfrak{g}$ acts transitively on $(M, g)$.

The following simple observation gives many examples of Killing vector fields of constant length on homogeneous Riemannian manifolds. Suppose that a Lie algebra $\mathfrak{g}$ of Killing vector fields is transitive on a Riemannian manifold $(M, g)$ and the Killing field $Z$ on $(M, g)$ commutes with $\mathfrak{g}$ (in particular, $Z$ is in the center of $\mathfrak{g}$ ), then $Z$ has a constant length on $(M, g)$. Indeed, for any $X \in \mathfrak{g}$ we have $X \cdot g(Z, Z)=$ $2 g([X, Z], Z)=0$. Since $\mathfrak{g}$ is transitive on $M$, we get $g(Z, Z)=$ const.

Example 1. Consider the irreducible symmetric space $M=S U(2 n) / S p(n), n \geq 2$. It is known that the subgroup $S U(2 n-1) \cdot S^{1} \subset S U(2 n)$ acts transitively on $M$, see 
e.g. [24] or [32]. Therefore, the Killing vector $Z$ generated by $S^{1}$, is a Killing field of constant length on $M=S U(2 n) / S p(n)$. The centralizer $\mathfrak{k}$ of $Z$ in $\mathfrak{g}_{1}$ is obviously $s u(2 n-1) \oplus \mathbb{R}$, i.e. $\left(\mathfrak{g}_{1}, \mathfrak{k}\right)=(s u(2 n), s u(2 n-1) \oplus \mathbb{R})$, see case 1$)$ in theorem 2.

Example 2. Consider the sphere $S^{2 n-1}, n \geq 2$, as the symmetric space $S^{2 n-1}=$ $S O(2 n) / S O(2 n-1)$. It is known that the subgroup $U(n)=S U(n) \cdot S^{1} \subset S O(2 n)$ acts transitively on $S^{2 n-1}$. Therefore, the Killing vector $Z$ generating $S^{1}$, is a Killing field of constant length on $S^{2 n-1}$. The centralizer $\mathfrak{k}$ of $Z$ in $\mathfrak{g}_{1}$ is $u(n)=s u(n) \oplus \mathbb{R}$, i.e. $\left(\mathfrak{g}_{1}, \mathfrak{k}\right)=($ so $(2 n)$, su $(n) \oplus \mathbb{R})$, see case 2$)$ in theorem 2.

Example 3. Consider the sphere $S^{4 n-1}, n \geq 2$, as the symmetric space $S^{4 n-1}=$ $S O(4 n) / S O(4 n-1)$. It is known that the subgroup $S p(n) \cdot S^{1} \subset S O(4 n)$ acts transitively on $S^{4 n-1}$. Therefore, the Killing vector $Z$ generated by $S^{1}$, is a Killing field of constant length on $S^{4 n-1}$. On the other hand, the centralizer $\mathfrak{k}$ of $Z$ in $\mathfrak{g}_{1}$ is $s u(2 n) \oplus \mathbb{R}$, since $s p(n) \oplus \mathbb{R} \subset \operatorname{su}(2 n) \oplus \mathbb{R}$ (see details e.g. in [10]). Therefore, $\left(\mathfrak{g}_{1}, \mathfrak{k}\right)=(\operatorname{so}(4 n)$, su $(2 n) \oplus \mathbb{R})$, see case 2$)$ in theorem 2 .

Note that the full connected isometry group of the sphere $S^{n-1}$ with the canonical Riemannian metric $g_{\text {can }}$ of constant curvature 1 , is $S O(n)$, but there are some subgroups $G$ of $S O(n)$ with transitive action on $S^{n-1}$. It is interesting that $S^{n-1}$ is $G$-Clifford-Wolf homogeneous for some of them: $S^{2 n-1}=S O(2 n) / S O(2 n-1)=$ $U(n) / U(n-1)$ is $S O(2 n)$-Clifford-Wolf homogeneous and $U(n)$-Clifford-Wolf homogeneous; $S^{4 n-1}=S p(n) / S p(n-1)=S p(n) \cdot S^{1} / S p(n-1) \cdot S^{1}=S U(2 n) / S U(2 n-1)$ is $S p(n)$-Clifford-Wolf homogeneous, $S p(n) \cdot S^{1}$-Clifford-Wolf homogeneous, and $S U(2 n)$ Clifford-Wolf homogeneous; $S^{7}=\operatorname{Spin}(7) / G_{2}$ is $\operatorname{Spin}(7)$-Clifford-Wolf homogeneous; $S^{15}=\operatorname{Spin}(9) / \operatorname{Spin}(7)$ is $\operatorname{Spin}(9)$-Clifford-Wolf homogeneous (see details in [10]). Every of these results gives an example of Killing vector field of constant length in the Lie algebra $\mathfrak{g}$ corresponded to the group $G$.

Example 4. There exists a Killing vector field of constant length $Z$ on $\left(S^{4 p-1}, g_{\text {can }}\right)$ such that $Z \in \operatorname{su}(2 p)$ and the centralizer $\mathfrak{k}$ of $Z$ in $s u(2 p)$ is $s u(p) \oplus s u(p) \oplus \mathbb{R}$, i.e. $\left(\mathfrak{g}_{1}, \mathfrak{k}\right)=(s u(2 p), s u(p) \oplus s u(p) \oplus \mathbb{R})$, see case 1$)$ in theorem 2 and proposition 12 in [10]. Note, that we may take $Z=\operatorname{diag}(\underbrace{\mathbf{i}, \ldots, \mathbf{i}}_{p}, \underbrace{-\mathbf{i}, \ldots,-\mathbf{i}}_{p}) \in \operatorname{su}(2 p)$.

Example 5. There exists a Killing vector field of constant length $Z$ on $\left(S^{2(p+q)-1}, g_{\text {can }}\right)$ such that $Z \in u(p+q)$ and the centralizer $\mathfrak{k}$ of $Z$ in $u(p+q)$ is $u(p) \oplus u(q)$, i.e. $\left(\mathfrak{g}_{1}, \mathfrak{k}\right)=(s u(p+q), s u(p) \oplus s u(q) \oplus \mathbb{R})$, see case 1$)$ in theorem 2 and proposition 12 in [10]. Note, that we may take $Z=\operatorname{diag}(\underbrace{\mathbf{i}, \ldots, \mathbf{i}}_{p}, \underbrace{-\mathbf{i}, \ldots,-\mathbf{i}}_{q}) \in u(p+q)$. Note also that the Lie algebra $u(p+q)$ has a 1-dimensional center, and $Z_{0}=0$ if and only if $p=q$, when we get the previous example. If $p \neq q$, then the Killing field $Z_{1}$ does not have a constant length on $\left(S^{2(p+q)-1}, g_{\text {can }}\right)$ by proposition 13 , see also proposition 14 .

Example 6. There exists a Killing vector field of constant length $Z$ on $\left(S^{7}, g_{\text {can }}\right)$ such that $Z \in \operatorname{spin}(7) \simeq \operatorname{so}(7)$ and the centralizer $\mathfrak{k}$ of $Z$ in $\operatorname{spin}(7) \simeq \operatorname{so}(7)$ is $\operatorname{so}(5) \oplus \mathbb{R}$, i.e. $\left(\mathfrak{g}_{1}, \mathfrak{k}\right)=($ so $(7)$, so $(5) \oplus \mathbb{R})$, see case 3$)$ in theorem 2 and remark 6 in $[10]$.

Example 7. There exists a Killing vector field of constant length $Z$ on $\left(S^{15}, g_{\text {can }}\right)$ such that $Z \in \operatorname{spin}(9)$ and the centralizer $\mathfrak{k}$ of $Z$ in $\operatorname{spin}(9) \simeq \operatorname{so}(9)$ is $\operatorname{so}(7) \oplus \mathbb{R}$, i.e. $\left(\mathfrak{g}_{1}, \mathfrak{k}\right)=($ so $(9)$, so $(7) \oplus \mathbb{R})$, see case 3$)$ in theorem 2 and proposition 20 in [10]. 
Example 8. There exists a Killing vector field of constant length $Z$ on $\left(S^{4 n-1}, g_{\text {can }}\right)$ such that $Z \in \operatorname{sp}(n)$ and the centralizer $\mathfrak{k}$ of $Z$ in $\operatorname{sp}(n)$ is $u(n)$, i.e. $\left(\mathfrak{g}_{1}, \mathfrak{k}\right)=$ $(\operatorname{sp}(n), s u(n) \oplus \mathbb{R})$, see case 4$)$ in theorem 2 and proposition 12 in [10]. Note, that we may take $Z=\operatorname{diag}(\mathbf{i}, \mathbf{i}, \ldots, \mathbf{i}) \in \operatorname{sp}(n)$.

\section{Preliminaries on Killing Vector fields of COnstant length}

Let $(M, g)$ be a connected Riemannian manifolds, $G$ be a connected Lie group acting isometrically on $(M, g)(x \mapsto a(x), x \in M, a \in G)$. Let $\mathfrak{g}$ be the Lie algebra of the group $G$, all elements of $\mathfrak{g}$ we identify with Killing fields on $(M, g)$ via (1). We recall some important properties of Killing vector fields, in particular, Killing fields of constant length on $(M, g)$. We also prove some useful results on Killing vector fields of constant length.

Lemma 1 (Lemma 3 in [8]). Let $X$ be a Killing vector field on a Riemannian manifold $(M, g)$. Then the following conditions are equivalent:

1) $X$ has constant length on $M$;

2) $\nabla_{X} X=0$ on $M$;

3) every integral curve of the field $X$ is a geodesic in $(M, g)$.

Lemma 2. If a Killing vector field $X \in \mathfrak{g}$ has constant length on $(M, g)$, then for any $Y, Z \in \mathfrak{g}$ the equalities

$$
\begin{aligned}
g([Y, X], X) & =0, \\
g([Z,[Y, X]], X)+g([Y, X],[Z, X]) & =0
\end{aligned}
$$

hold at every point of $M$. If $G$ acts on $(M, g)$ transitively, then condition (3) implies that $X$ has constant length. Moreover, the condition (4) also implies that $X$ has constant length for compact $M$ and transitive $G$.

Proof. If $g(X, X)=$ const, then $2 g([Y, X], X)=Y \cdot g(X, X)=0$ at every point of $M$ for every $Y \in \mathfrak{g}$, that proves (3). From this we have $0=Z \cdot g([Y, X], X)=$ $g([Z,[Y, X]], X)+g([Y, X],[Z, X])$ for any $Z \in \mathfrak{g}$, that proves (4).

In the case of transitive action, we obviously get $g(X, X)=$ const from the equality $Y \cdot g(X, X)=2 g([X, Y], X)=0, Y \in \mathfrak{g}$.

Note also that in the case of transitive action the condition (4) means that for any $Y \in \mathfrak{g}$ we have $g([Y, X], X)=C=$ const on $M$. If $M$ is compact then there is $x \in M$ where $g(X, X)$ has its maximal value. Obviously, that $g_{x}([Y, X], X)=0$. Therefore, $C=0$ and $X$ has constant length by the previous assertion.

Lemma 3 (see e.g. lemma 7.27 in [4]). For Killing vector fields $X, Y, Z$ on a Riemannian manifold $(M, g)$, the equality

$2 g\left(\nabla_{X} Y, Z\right)=g([X, Y], Z)+g([X, Z], Y)+g(X,[Y, Z])=g([X, Y], Z)-Z \cdot g(X, Y)$ holds. In particular, $\nabla_{X} Y=\frac{1}{2}[X, Y]$, if $g(X, Y)=$ const and $(M, g)$ is homogeneous.

Lemma 4. Let $X$ be a Killing field and let $Y$ be a Killing field of constant length on a Riemannian manifold $(M, g)$. Then the formula $R(X, Y) Y=-\nabla_{Y} \nabla_{Y} X$ holds on $M$ $\left(R(X, Z):=\nabla_{X} \nabla_{Z}-\nabla_{Z} \nabla_{X}-\nabla_{[X, Z]}\right)$.

Proof. All integral curve of the field $Y$ are geodesics by lemma 1. On the other hand, the restriction of the Killing field $X$ on any geodesic is an Jacobi field (see e.g. proposition 1.3 of chapter VIII in [18]). Hence, $\nabla_{Y} \nabla_{Y} X+R(X, Y) Y=0$ on $M$. 
We will use the identification (1) of elements of Lie algebras $\mathfrak{g}$ of $G$ with corresponding Killing vector fields on $(M, g)$ in the proof of the following

Lemma 5. Let $X, Y \in \mathfrak{g}$ be such that $g(X, Y)=C=$ const on $M$. Then for every inner automorphism $A$ of $\mathfrak{g}$ we get $g(A(X), A(Y))=C$ at every point of $M$. In particular, if $X \in \mathfrak{g}$ has constant length on $(M, g)$, then $A(X)$ has the same property.

Proof. If $L_{a}: M \rightarrow M$ is the action of $a \in G$ on $M$, then for any $U \in \mathfrak{g}$ we have

$$
\begin{aligned}
d L_{a}(\widetilde{U}(x))= & \left.\frac{d}{d t}(a \exp (t U))(x)\right|_{t=0}=\left.\frac{d}{d t}\left(a \exp (t U) a^{-1}\right)(a(x))\right|_{t=0}= \\
& \left.\frac{d}{d t}(\exp (\operatorname{Ad}(a)(U) t+o(t)))(a(x))\right|_{t=0}=\widetilde{\operatorname{Ad}(a)(U)}(a(x)) .
\end{aligned}
$$

Since $L_{a}$ is an isometry of $(M, g)$, then

$$
g_{x}(\tilde{X}, \tilde{Y})=g_{L_{a}(x)}\left(d L_{a}(\tilde{X}), d L_{a}(\tilde{Y})\right)=g_{a(x)}(\widetilde{\operatorname{Ad}(a)(X)}, \widetilde{\operatorname{Ad}(a)(Y)})
$$

at every point $x \in M$. Recall that the inner automorphism group of $\mathfrak{g}$ coincides with the adjoint group of $G$, therefore we get the lemma.

\section{Some Algebraic lemmas}

A Lie algebra $\mathfrak{g}$ is called compact, if it is a Lie algebra of some compact Lie group. Any such Lie algebra admits an ad( $\mathfrak{g})$-invariant inner product. The following lemma is known in the literature, but we include its proof for completeness.

Lemma 6. Suppose that $\mathfrak{h}$ is a subalgebra of a compact Lie algebra $\mathfrak{g}$, and $\mathfrak{p}$ is an $\langle\cdot, \cdot\rangle$-orthogonal complement to $\mathfrak{h}$ in $\mathfrak{g}$, where $\langle\cdot, \cdot\rangle$ is an ad $(\mathfrak{g})$-invariant inner product on $\mathfrak{g}$. Then the sets $\mathfrak{p}+[\mathfrak{p}, \mathfrak{p}]$ and $\mathfrak{u}:=\{Z \in \mathfrak{h} \mid[Z, \mathfrak{p}]=0\}$ are ideals in $\mathfrak{g}$.

Proof. Since $[\mathfrak{u}, \mathfrak{h}] \subset \mathfrak{h}$ and $\mathfrak{p}$ is $\operatorname{ad}(\mathfrak{h})$-invariant, we get

$$
[\mathfrak{p},[\mathfrak{u}, \mathfrak{h}]] \subset[[\mathfrak{p}, \mathfrak{u}], \mathfrak{h}]]+[\mathfrak{u},[\mathfrak{p}, \mathfrak{h}]]=0,
$$

hence $[\mathfrak{u}, \mathfrak{h}] \subset \mathfrak{u}$. Since $[\mathfrak{u}, \mathfrak{p}]=0$, we get that $\mathfrak{u}$ is an ideals in $\mathfrak{g}$. It is easy to check that $\mathfrak{p}+[\mathfrak{p}, \mathfrak{p}]$ is the complementary ideal to $\mathfrak{u}$ in $\mathfrak{g}$. Indeed, for $Z \in \mathfrak{h}$ the condition $\langle Z,[\mathfrak{p}, \mathfrak{p}]\rangle=0$ is equivalent to every of the conditions $\langle[Z, \mathfrak{p}], \mathfrak{p}\rangle=0$ and $[Z, \mathfrak{p}]=0$.

We will need also the following generalization of the previous lemma (compare e.g. with the proof of Theorem 2.1 in [28]).

Lemma 7. Let $\mathfrak{g}$ be a compact Lie algebra, $\langle\cdot, \cdot\rangle$ be an $\operatorname{ad}(\mathfrak{g})$-invariant inner product on $\mathfrak{g}, \mathfrak{h}$ be a subalgebra of $\mathfrak{g}$. Suppose that the $\langle\cdot, \cdot \cdot\rangle$-orthogonal complement $\mathfrak{p}$ to $\mathfrak{h}$ in $\mathfrak{g}$ is of the type

$$
\mathfrak{p}=\mathfrak{p}_{1} \oplus \mathfrak{p}_{2} \oplus \cdots \oplus \mathfrak{p}_{k}
$$

where every $\mathfrak{p}_{i}$ is $\operatorname{ad}(\mathfrak{h})$-invariant and $\operatorname{ad}(\mathfrak{h})$-irreducible, $\left\langle\mathfrak{p}_{i}, \mathfrak{p}_{j}\right\rangle=0$ and $\left[\mathfrak{p}_{i}, \mathfrak{p}_{j}\right]=0$ for $i \neq j$. For $1 \leq i \leq k$ put $\mathfrak{h}_{i}:=\left(\mathfrak{p}_{i}+\left[\mathfrak{p}_{i}, \mathfrak{p}_{i}\right]\right) \cap \mathfrak{h}$ and $\mathfrak{g}_{i}:=\mathfrak{h}_{i} \oplus \mathfrak{p}_{i}$. Then the following assertions hold:

1) $\mathfrak{g}_{i}=\mathfrak{p}_{i}+\left[\mathfrak{p}_{i}, \mathfrak{p}_{i}\right]$ for every $1 \leq i \leq k$;

2) every $\mathfrak{g}_{i}$ is an ideal in $\mathfrak{g}$;

3) $\left\langle\mathfrak{g}_{i}, \mathfrak{g}_{j}\right\rangle=0$ for $i \neq j$;

4) every pair $\left(\mathfrak{g}_{i}, \mathfrak{h}_{i}\right)$ is effective and isotropy irreducible;

5) $\mathfrak{u}$, the $\langle\cdot, \cdot\rangle$-orthogonal complement to $\bigoplus_{i} \mathfrak{g}_{i}$ in $\mathfrak{g}$, is an ideal in $\mathfrak{g}$;

6) $\mathfrak{g}=\mathfrak{u} \oplus \mathfrak{g}_{1} \oplus \mathfrak{g}_{2} \oplus \cdots \oplus \mathfrak{g}_{k}, \mathfrak{h}=\mathfrak{u} \oplus \mathfrak{h}_{1} \oplus \mathfrak{h}_{2} \oplus \cdots \oplus \mathfrak{h}_{k}$. 
Proof. At first, we prove that $\mathfrak{g}_{i}=\mathfrak{p}_{i}+\left[\mathfrak{p}_{i}, \mathfrak{p}_{i}\right]$ for every $1 \leq i \leq k$. Consider any $j \neq i, 1 \leq j \leq k$. For every $X, Z \in \mathfrak{p}_{i}$ and $Y \in \mathfrak{p}_{j}$, we have $\langle[Z, X], Y\rangle=$ $-\langle X,[Z, Y]\rangle=0$, since $\left[\mathfrak{p}_{i}, \mathfrak{p}_{j}\right]=0$ and $\langle\cdot, \cdot\rangle$ is ad $(\mathfrak{g})$-invariant. Hence, $\left\langle\left[\mathfrak{p}_{i}, \mathfrak{p}_{i}\right], \mathfrak{p}_{j}\right\rangle=0$, that implies $\operatorname{pr}_{\mathfrak{p}}\left(\left[\mathfrak{p}_{i}, \mathfrak{p}_{i}\right]\right) \subset \mathfrak{p}_{i}$ and, therefore, $\mathfrak{g}_{i}=\mathfrak{p}_{i}+\left[\mathfrak{p}_{i}, \mathfrak{p}_{i}\right]$.

Since $\left[\mathfrak{p}_{i}, \mathfrak{p}_{j}\right]=0$, then by the Jacobi equality we get $\left[\mathfrak{p}_{j},\left[\mathfrak{p}_{i}, \mathfrak{p}_{i}\right]\right]=0$. Therefore, $\left\langle\left[\mathfrak{p}_{j},\left[\mathfrak{p}_{i}, \mathfrak{p}_{i}\right]\right], \mathfrak{g}\right\rangle=0$ and $\left\langle\left[\mathfrak{p}_{i}, \mathfrak{p}_{i}\right],\left[\mathfrak{p}_{j}, \mathfrak{g}\right]\right\rangle=0$ by the ad $(\mathfrak{g})$-invariance of $\langle\cdot, \cdot\rangle$. From $\left\langle\left[\mathfrak{p}_{i}, \mathfrak{p}_{i}\right],\left[\mathfrak{p}_{j}, \mathfrak{g}\right]\right\rangle=0$ we get $\left\langle\left[\mathfrak{p}_{i}, \mathfrak{p}_{i}\right],\left[\mathfrak{p}_{j}, \mathfrak{p}_{j}\right]\right\rangle=0$ in particular. This equality together with the equalities $\left\langle\left[\mathfrak{p}_{i}, \mathfrak{p}_{i}\right], \mathfrak{p}_{j}\right\rangle=0,\left\langle\left[\mathfrak{p}_{j}, \mathfrak{p}_{j}\right], \mathfrak{p}_{i}\right\rangle=0$, and $\left\langle\mathfrak{p}_{i}, \mathfrak{p}_{j}\right\rangle=0$ imply $\left\langle\mathfrak{g}_{i}, \mathfrak{g}_{j}\right\rangle=0$, since $\mathfrak{g}_{i}=\mathfrak{p}_{i}+\left[\mathfrak{p}_{i}, \mathfrak{p}_{i}\right]$ and $\mathfrak{g}_{j}=\mathfrak{p}_{j}+\left[\mathfrak{p}_{j}, \mathfrak{p}_{j}\right]$.

Since $\left[\mathfrak{p}_{j},\left[\mathfrak{p}_{i}, \mathfrak{p}_{i}\right]\right]=0$ and $\left[\mathfrak{p}_{j}, \mathfrak{p}_{i}\right]=0$ for $i \neq j$, then $\left[\mathfrak{p}_{j}, \mathfrak{g}_{i}\right]=0,0=\left[\mathfrak{p}_{j},\left[\mathfrak{g}_{i}, \mathfrak{p}_{j}\right]\right]=$ $\left[\mathfrak{g}_{i},\left[\mathfrak{p}_{j}, \mathfrak{p}_{j}\right]\right]$, and $\left[\mathfrak{g}_{i}, \mathfrak{g}_{j}\right]=0$.

It is clear that $\mathfrak{u} \subset \mathfrak{h}$. Since $\left\langle\mathfrak{u},\left[\mathfrak{p}_{i}, \mathfrak{p}_{i}\right]\right\rangle=0$ for any $i$, then $\left\langle\left[\mathfrak{p}_{i}, \mathfrak{u}\right], \mathfrak{p}_{i}\right\rangle=0$, which means, that $[\mathfrak{u}, \mathfrak{p}]=0$. It is easy to check that $\mathfrak{u}=\{Z \in \mathfrak{h} \mid[Z, \mathfrak{p}]=0\}$. By the Lemma 6 we get that $\mathfrak{u}$ is an ideal in $\mathfrak{g}$. Now, all assertions of the lemma are clear.

At first, we prove that $\mathfrak{g}_{i}=\mathfrak{p}_{i}+\left[\mathfrak{p}_{i}, \mathfrak{p}_{i}\right]$ for every $1 \leq i \leq k$. Consider any $j \neq i$, $1 \leq j \leq k$. Since $\left[\mathfrak{p}_{i}, \mathfrak{p}_{j}\right]=0$, then by the Jacobi equality we get $\left[\mathfrak{p}_{j},\left[\mathfrak{p}_{i}, \mathfrak{p}_{i}\right]\right]=0$. Therefore, $\left\langle\left[\mathfrak{p}_{j},\left[\mathfrak{p}_{i}, \mathfrak{p}_{i}\right]\right], \mathfrak{g}\right\rangle=0$ and $\left\langle\left[\mathfrak{p}_{i}, \mathfrak{p}_{i}\right],\left[\mathfrak{p}_{j}, \mathfrak{g}\right]\right\rangle=0$ by the $\operatorname{ad}(\mathfrak{h})$-invariance of $\langle\cdot, \cdot\rangle$. Since $\mathfrak{p}_{j}$ is $\operatorname{ad}(\mathfrak{h})$-irreducible, we get $\left\langle\left[\mathfrak{p}_{i}, \mathfrak{p}_{i}\right],\left[\mathfrak{p}_{j}, \mathfrak{h}\right]\right\rangle=\left\langle\left[\mathfrak{p}_{i}, \mathfrak{p}_{i}\right], \mathfrak{p}_{j}\right\rangle=0$. This means that $\operatorname{pr}_{\mathfrak{p}}\left(\left[\mathfrak{p}_{i}, \mathfrak{p}_{i}\right]\right) \subset \mathfrak{p}_{i}$ and, therefore, $\mathfrak{g}_{i}=\mathfrak{p}_{i}+\left[\mathfrak{p}_{i}, \mathfrak{p}_{i}\right]$.

From $\left\langle\left[\mathfrak{p}_{i}, \mathfrak{p}_{i}\right],\left[\mathfrak{p}_{j}, \mathfrak{g}\right]\right\rangle=0$ we get also that $\left\langle\left[\mathfrak{p}_{i}, \mathfrak{p}_{i}\right],\left[\mathfrak{p}_{j}, \mathfrak{p}_{j}\right]\right\rangle=0$. This equality together with $\left\langle\left[\mathfrak{p}_{i}, \mathfrak{p}_{i}\right], \mathfrak{p}_{j}\right\rangle=0$ imply $\left\langle\left[\mathfrak{p}_{i}, \mathfrak{p}_{i}\right], \mathfrak{g}_{j}\right\rangle=0$.

Further, $\left\langle\left[\mathfrak{p}_{i}, \mathfrak{p}_{j}\right], \mathfrak{g}\right\rangle=0$, then by the $\operatorname{ad}(\mathfrak{h})$-invariance of $\langle\cdot, \cdot\rangle$ we get $\left\langle\mathfrak{p}_{i},\left[\mathfrak{g}, \mathfrak{p}_{j}\right]\right\rangle=0$, in particular, $\left\langle\mathfrak{p}_{i}, \mathfrak{g}_{j}\right\rangle=0$. This equation and $\left\langle\left[\mathfrak{p}_{i}, \mathfrak{p}_{i}\right], \mathfrak{g}_{j}\right\rangle=0$ imply $\left\langle\mathfrak{g}_{i}, \mathfrak{g}_{j}\right\rangle=0$ for $i \neq j$, since $\mathfrak{g}_{i}=\mathfrak{p}_{i}+\left[\mathfrak{p}_{i}, \mathfrak{p}_{i}\right]$.

Since $\left[\mathfrak{p}_{j},\left[\mathfrak{p}_{i}, \mathfrak{p}_{i}\right]\right]=0$ for $i \neq j$, then $\left[\mathfrak{p}_{j}, \mathfrak{g}_{i}\right]=0,0=\left[\mathfrak{p}_{j},\left[\mathfrak{g}_{i}, \mathfrak{p}_{j}\right]\right]=\left[\mathfrak{g}_{i},\left[\mathfrak{p}_{j}, \mathfrak{p}_{j}\right]\right]$ and $\left[\mathfrak{g}_{i}, \mathfrak{g}_{j}\right]=0$.

It is clear that $\mathfrak{u} \subset \mathfrak{h}$. Since $\left\langle\mathfrak{u},\left[\mathfrak{p}_{i}, \mathfrak{p}_{i}\right]\right\rangle=0$ for any $i$, then $\left\langle\left[\mathfrak{p}_{i}, \mathfrak{u}\right], \mathfrak{p}_{i}\right\rangle=0$, which means, that $[\mathfrak{u}, \mathfrak{p}]=0$. It is easy to check that $\mathfrak{u}=\{Z \in \mathfrak{h} \mid[Z, \mathfrak{p}]=0\}$. By the Lemma 6 we get that $\mathfrak{u}$ is an ideal in $\mathfrak{g}$. Now, all assertions of the lemma are clear.

Let us consider any compact Lie algebra $\mathfrak{g}$ with $\operatorname{ad}(\mathfrak{g})$-invariant inner product $\langle\cdot, \cdot\rangle$. Take any $Z \in \mathfrak{g}$ and consider the operator $L_{Z}: \mathfrak{g} \rightarrow \mathfrak{g}, L_{Z}(X)=[Z, X]$. This operator is skew-symmetric, but $L_{Z}^{2}$ is a symmetric operator on $\mathfrak{g}$ with respect to $\langle\cdot, \cdot\rangle$. Put $\mathfrak{m}_{0}=\operatorname{Ker} L_{Z}=\{Y \in \mathfrak{g} \mid[Z, Y]=0\}$ and consider all non-zero eigenvalues of the operator $L_{Z}^{2}:-\lambda_{i}^{2}, i=1, \ldots, s$, where $0<\lambda_{1}<\lambda_{1}<\cdots<\lambda_{s}$, and the corresponding eigenspaces $\mathfrak{m}_{\lambda_{i}}=\left\{Y \in \mathfrak{g} \mid L_{Z}^{2}(Y)=[Z,[Z, Y]]=-\lambda_{i}^{2} Y\right\}$. It is clear that $\mathfrak{m}_{0}$ is a Lie subalgebra of $\mathfrak{g}$ of maximal rank and

$$
\mathfrak{m}:=\mathfrak{m}_{\lambda_{1}} \oplus \mathfrak{m}_{\lambda_{2}} \oplus \cdots \oplus \mathfrak{m}_{\lambda_{s}}=\operatorname{Im} L_{Z}
$$

Note that this decomposition and the decomposition $\mathfrak{g}=\mathfrak{m}_{0} \oplus \mathfrak{m}$ are $\langle\cdot, \cdot\rangle$-orthogonal. It follows from the simple observation: If $P \subset \mathfrak{g}$ is invariant subspace of the operator $L_{Z}^{2}$, then its $\langle\cdot, \cdot\rangle$-orthogonal complement $P^{\perp}$ is also invariant subspace of $L_{Z}^{2}$.

We will need the following simple

Lemma 8. For any $X \in \mathfrak{m}_{0}$ and $Y \in \mathfrak{m}_{\alpha}$ we have $[X, Y] \in \mathfrak{m}_{\alpha}$, i.e. every $\mathfrak{m}_{\alpha}$ is $\operatorname{ad}\left(\mathfrak{m}_{0}\right)$-invariant. In particular, $\left[Z, \mathfrak{m}_{\alpha}\right] \subset \mathfrak{m}_{\alpha}$.

Proof. We have $L_{Z}([X, Y])=[X,[Z, Y]]$ and $L_{Z}^{2}([X, Y])=[X,[Z,[Z, Y]]]=$ $-\lambda_{i}^{2}[X, Y]$. 
Now we define a linear operator $\sigma: \mathfrak{m} \rightarrow \mathfrak{m}$ as follows:

$$
\sigma(Y)=\frac{1}{\lambda_{i}}[Z, Y], \quad Y \in \mathfrak{m}_{\lambda_{i}}
$$

In fact, this operator define a complex structure on a flag manifold $G / C_{G}(Z)$, where $C_{G}(Z)$ is a centralizer of $Z$ in the group $G$ (see e.g. chapter 8 in [4]).

For any $X, Y \in \mathfrak{m}$ we define also

$$
[X, Y]^{+}:=\frac{1}{2}([X, Y]-[\sigma(X), \sigma(Y)]), \quad[X, Y]^{-}:=\frac{1}{2}([X, Y]+[\sigma(X), \sigma(Y)]) .
$$

Obviously, $[X, Y]=[X, Y]^{+}+[X, Y]^{-}$.

Proposition 1. In the above notation, $\sigma\left(\mathfrak{m}_{\alpha}\right) \subset \mathfrak{m}_{\alpha}$ for all $\alpha$ and $\sigma(\sigma(Y))=-Y$ for all $Y \in \mathfrak{m}$. If $U \in \mathfrak{m}_{\alpha}, V \in \mathfrak{m}_{\beta}$, then

$$
[U, V]^{+} \in \mathfrak{m}_{\alpha+\beta}, \quad[U, V]^{-} \in \mathfrak{m}_{|\alpha-\beta|} .
$$

Proof. The first assertion follows from lemma 8 , the equality $\sigma^{2}=-$ Id is obvious. By this equality and definitions we have

$$
\begin{array}{r}
L_{Z}([U, V])=[Z,[U, V]]=[[Z, U], V]+[U,[Z, V]]=\alpha[\sigma(U), V]+\beta[U, \sigma(V)], \\
L_{Z}([\sigma(U), \sigma(V)])=[[Z, \sigma(U)], \sigma(V)]+[\sigma(U),[Z, \sigma(V)]]=-\alpha[U, \sigma(V)]-\beta[\sigma(U), V], \\
L_{Z}^{2}([U, V])=[Z,[Z,[U, V]]]=-\left(\alpha^{2}+\beta^{2}\right)[U, V]+2 \alpha \beta[\sigma(U), \sigma(V)], \\
L_{Z}^{2}([\sigma(U), \sigma(V)])=[Z,[Z,[\sigma(U), \sigma(V)]]]=-\left(\alpha^{2}+\beta^{2}\right)[\sigma(U), \sigma(V)]+2 \alpha \beta[U, V] .
\end{array}
$$

Hence, we get $L_{Z}^{2}([U, V]-[\sigma(U), \sigma(V)])=-(\alpha+\beta)^{2}([U, V]-[\sigma(U), \sigma(V)])$ and $L_{Z}^{2}([U, V]+[\sigma(U), \sigma(V)])=-(\alpha-\beta)^{2}([U, V]+[\sigma(U), \sigma(V)])$.

Note that $\operatorname{rk}\left(\mathfrak{m}_{0}\right)=\operatorname{rk}(\mathfrak{g})$. This means that there is a unique (up to order of summands) $\langle\cdot, \cdot\rangle$-orthogonal ad $\left(\mathfrak{m}_{0}\right)$-invariant decomposition of $\mathfrak{m}$ into ad $\left(\mathfrak{m}_{0}\right)$-irreducible summands (see e.g. theorem 5.3 in [19])

$$
\mathfrak{m}=\mathfrak{p}_{1} \oplus \mathfrak{p}_{2} \oplus \cdots \oplus \mathfrak{p}_{t}
$$

Note, that every $\mathfrak{p}_{i}, 1 \leq i \leq t$, is a subspace of a suitable $\mathfrak{m}_{\lambda_{j}}, 1 \leq j \leq s$. Note also that every $\mathfrak{p}_{i}$ is invariant under the operator $\sigma$, because $Z \in \mathfrak{m}_{0}$.

\section{Root SpaCE DeComposition For Simple COMPACT Lie Algebras}

We give here some information on the root system of a compact simple Lie algebra $(\mathfrak{g},\langle\cdot, \cdot\rangle=-B)$ with the Killing form $B$, that can be found e.g. in $[12,17,26,32]$.

Let us fix a Cartan subalgebra $\mathfrak{t}$ (that is maximal abelian subalgebra) of Lie algebra $\mathfrak{g}$. There is a set $\Delta$ (root system) of (non-zero) real-valued linear form $\alpha \in \mathfrak{t}^{*}$ on the Cartan subalgebra $\mathfrak{t}$, that are called roots. Let us consider some positive root system $\Delta^{+} \subset \Delta$. Recall that for any $\alpha \in \Delta$ exactly one of the roots $\pm \alpha$ is positive (we denote it by $|\alpha|)$. The Lie algebra $\mathfrak{g}$ admits a direct $\langle\cdot, \cdot\rangle$-orthogonal decomposition

$$
\mathfrak{g}=\mathfrak{t} \oplus \bigoplus_{\alpha \in \Delta^{+}} \mathfrak{v}_{\alpha}
$$

into vector subspaces, where each subspace $\mathfrak{v}_{\alpha}$ is 2-dimensional and ad(t)-invariant. Using the restriction (of non-degenerate) inner product $\langle\cdot, \cdot\rangle$ to $\mathfrak{t}$, we will naturally identify $\alpha$ with some vector in $\mathfrak{t}$. Note that $\left[\mathfrak{v}_{\alpha}, \mathfrak{v}_{\alpha}\right]$ is one-dimensional subalgebra in $\mathfrak{t}$ spanned on the root $\alpha$, and $\left[\mathfrak{v}_{\alpha}, \mathfrak{v}_{\alpha}\right] \oplus \mathfrak{v}_{\alpha}$ is a Lie algebra isomorphic to $s u(2)$. The 
vector subspaces $\mathfrak{v}_{\alpha}, \alpha \in \Delta^{+}$, admit bases $\left\{U_{\alpha}, V_{\alpha}\right\}$, such that $\left\langle U_{\alpha}, U_{\alpha}\right\rangle=\left\langle V_{\alpha}, V_{\alpha}\right\rangle=1$, $\left\langle U_{\alpha}, V_{\alpha}\right\rangle=0$ and

$$
\left[H, U_{\alpha}\right]=\langle\alpha, H\rangle V_{\alpha}, \quad\left[H, V_{\alpha}\right]=-\langle\alpha, H\rangle U_{\alpha}, \quad \forall H \in \mathfrak{t}, \quad\left[U_{\alpha}, V_{\alpha}\right]=\alpha .
$$

Note also, that $\left[\mathfrak{v}_{\alpha}, \mathfrak{v}_{\beta}\right]=\mathfrak{v}_{\alpha+\beta}+\mathfrak{v}_{|\alpha-\beta|}$, assuming $\mathfrak{v}_{\gamma}:=\{0\}$ for $\gamma \notin \Delta^{+}$.

For a positive root system $\Delta^{+}$the (closed) Weyl chamber is defined by the equality

$$
C=C\left(\Delta^{+}\right):=\left\{H \in \mathfrak{t} \mid\langle\alpha, H\rangle \geq 0 \forall \alpha \in \Delta^{+}\right\} .
$$

Recall some important properties of the Weyl group $W=W(\mathfrak{t})$ of the Lie algebra $\mathfrak{g}$, that acts on the Cartan subalgebra $t$.

(i) For every root $\alpha \in \Delta \subset \mathfrak{t}$ the Weyl group $W$ contains the orthogonal reflection $\varphi_{\alpha}$ in the plane $P_{\alpha}$, which is orthogonal to the root $\alpha$ with respect to $\langle\cdot, \cdot\rangle$. It is easy to see that $\varphi_{\alpha}(H)=H-2 \frac{\langle H, \alpha\rangle}{\langle\alpha, \alpha\rangle} \alpha, H \in \mathfrak{t}$.

(ii) Reflections from (i) generate $W$.

(iii) The root system $\Delta$ is invariant under the action of the Weyl group $W$.

(iv) $W$ acts irreducible on $\mathfrak{t}$ and simply transitively on the set of positive root systems. For any $H \in \mathfrak{t}$, there is $w \in W$, such that $w(H) \in C\left(\Delta^{+}\right)$.

(v) For any $X \in \mathfrak{g}$, there is an inner automorphism $\psi$ of $\mathfrak{g}$ such that $\psi(X) \in \mathfrak{t}$. For any $w \in W$, there is an inner automorphism $\eta$ of $\mathfrak{g}$, such that $\mathfrak{t}$ is stable under $\eta$, and the restriction of $\eta$ to $\mathfrak{t}$ coincides with $w$.

(vi) The Weyl group $W$ acts transitively on the set of positive roots of fixed length.

For a given positive root system $\Delta^{+}$, we denote by $\Pi=\left\{\pi_{1}, \ldots, \pi_{r}\right\}$ the set of simple root in $\Delta^{+}, r=\operatorname{rk}(\mathfrak{g})$. Note that every $\alpha \in \Delta^{+}$there is a unique decomposition $\alpha=\sum_{i=1}^{r} a_{i} \pi_{i}$ with non-negative integer $a_{i}$. There is the maximal root $\beta \in \Delta^{+}$that is characterized by the fact that $\beta-\alpha$ is a non-negative linear combination of roots in $\Delta^{+}$for all other positive roots $\alpha$. We will denote this root by $\alpha_{\max }$.

We list below some important properties of some root systems which we will use later (see [11]). In all cases $(\mathfrak{t},\langle\cdot, \cdot\rangle)$ is identified with a suitable subspace of Euclidean space $\mathbb{R}^{n}$ with the orthonormal basis $\left\{e_{i}\right\}$.

Case 1. $A_{l}=s u(l+1), l \geq 1: \mathfrak{t}=\left\{\left(x_{1}, x_{2}, \ldots, x_{l+1}\right) \in \mathbb{R}^{l+1} \mid x_{1}+x_{2}+\cdots+x_{l+1}=0\right\}$,

$$
\begin{array}{r}
\Delta=\left\{e_{i}-e_{j} \mid i \neq j, 1 \leq i, j \leq l+1\right\}, \\
\Pi=\left\{\pi_{i}=e_{i}-e_{i+1} \mid i=1, \ldots, l\right\}, \quad \alpha_{\max }=\sum_{i=1}^{l} \pi_{i} .
\end{array}
$$

Case 2. $B_{l}=s o(2 l+1), l \geq 2: \mathfrak{t}=\mathbb{R}^{l}$,

$$
\begin{array}{r}
\Delta=\left\{ \pm e_{i} \mid i=1,2, \ldots, l\right\} \cup\left\{ \pm e_{i} \pm e_{j} \mid 1 \leq i<j \leq l\right\}, \\
\Pi=\left\{\pi_{i}=e_{i}-e_{i+1} \mid i=1, \ldots, l-1\right\} \cup\left\{\pi_{l}=e_{l}\right\}, \quad \alpha_{\max }=\pi_{1}+2 \sum_{i=2}^{l} \pi_{i} .
\end{array}
$$

Case 3. $C_{l}=\operatorname{sp}(l), l \geq 2: \mathfrak{t}=\mathbb{R}^{l}$,

$$
\begin{array}{r}
\Delta=\left\{ \pm 2 e_{i} \mid i=1,2, \ldots, l\right\} \cup\left\{ \pm e_{i} \pm e_{j} \mid 1 \leq i<j \leq l\right\}, \\
\Pi=\left\{\pi_{i}=e_{i}-e_{i+1} \mid i=1, \ldots, l-1\right\} \cup\left\{\pi_{l}=2 e_{l}\right\}, \quad \alpha_{\max }=2 \sum_{i=1}^{l-1} \pi_{i}+\pi_{l} .
\end{array}
$$


Case 4. $D_{l}=s o(2 l), l \geq 3: \mathfrak{t}=\mathbb{R}^{l}$,

$$
\begin{array}{r}
\Delta=\left\{ \pm e_{i} \pm e_{j} \mid 1 \leq i<j \leq l\right\}, \\
\Pi=\left\{\pi_{i}=e_{i}-e_{i+1} \mid i=1, \ldots, l-1\right\} \cup\left\{\pi_{l}=e_{l-1}+e_{l}\right\}, \\
\alpha_{\max }=\pi_{1}+2 \sum_{i=2}^{l-2} \pi_{i}+\pi_{l-1}+\pi_{l} .
\end{array}
$$

Case 5. $e_{6}: \mathfrak{t}=\left\{\left(x_{1}, x_{2}, \ldots, x_{8}\right) \in \mathbb{R}^{8} \mid x_{6}=x_{7}=-x_{8}\right\}$,

$$
\begin{array}{r}
\Delta=\left\{ \pm e_{i} \pm e_{j} \mid 1 \leq i<j \leq 5\right\} \cup \\
\left\{ \pm \frac{1}{2}\left(e_{8}-e_{7}-e_{6}+\sum_{i=1}^{5}(-1)^{v_{i}} e_{i}\right) \mid v_{i} \in\{0,1\}, \sum_{i=1}^{5} v_{i} \text { is even }\right\}, \\
\Pi=\left\{\pi_{1}=\frac{1}{2}\left(e_{1}+e_{8}\right)-\frac{1}{2} \sum_{i=2}^{7} e_{i}\right\} \cup\left\{\pi_{2}=e_{1}+e_{2}\right\} \cup\left\{\pi_{i}=e_{i-1}-e_{i-2} \mid i=3, \ldots, 6\right\}, \\
\alpha_{\max }=\pi_{1}+2 \pi_{2}+2 \pi_{3}+3 \pi_{4}+2 \pi_{5}+\pi_{6} .
\end{array}
$$

Case 6. $e_{7}: \mathfrak{t}=\left\{x \in \mathbb{R}^{8} \mid x\right.$ orthogonal to $\left.e_{7}+e_{8}\right\}$,

$$
\begin{array}{r}
\Delta=\left\{ \pm e_{i} \pm e_{j} \mid 1 \leq i<j \leq 6\right\} \cup\left\{ \pm\left(e_{7}-e_{8}\right)\right\} \cup \\
\left\{ \pm \frac{1}{2}\left(e_{7}-e_{8}+\sum_{i=1}^{6}(-1)^{v_{i}} e_{i}\right) \mid v_{i} \in\{0,1\}, \sum_{i=1}^{6} v_{i} \text { is odd }\right\}, \\
\Pi=\left\{\pi_{1}=\frac{1}{2}\left(e_{1}+e_{8}\right)-\frac{1}{2} \sum_{i=2}^{7} e_{i}\right\} \cup\left\{\pi_{2}=e_{1}+e_{2}\right\} \cup\left\{\pi_{i}=e_{i-1}-e_{i-2} \mid i=3, \ldots, 7\right\}, \\
\alpha_{\max }=2 \pi_{1}+2 \pi_{2}+3 \pi_{3}+4 \pi_{4}+3 \pi_{5}+2 \pi_{6}+\pi_{7} .
\end{array}
$$

Recall that a simple root $\pi_{i}$ is called non-compact if, for every $\alpha \in \Delta$ either $\alpha$ is of the form $\alpha= \pm \sum_{j \neq i} a_{j} \pi_{j}$, or $\alpha$ is of the form $\alpha= \pm\left(\pi_{i}+\sum_{j \neq i} a_{j} \pi_{j}\right)$, where $a_{i} \geq 0$. The Lie algebras from cases 1-6 are exactly simple Lie algebras that have non-compact roots. These are the roots with coefficient 1 in the decomposition of the maximal root $\alpha_{\max }$. Non-compact root are closely related to Hermitian symmetric spaces, see [31] or [32] for a comprehensive description.

\section{Killing FieldS OF CONSTANT LENGTH ON COMPACT HOMOGENEOUS RIEMANNIAN MANIFOLDS}

In this section we suppose that $(M, g)$ is a compact Riemannian manifold, and $G$ is a connected compact transitive isometry group of $(M, g)$. We fix any $\operatorname{Ad}(G)$-invariant inner product $\langle\cdot, \cdot\rangle$ on the Lie algebra $\mathfrak{g}$.

Proposition 2. For a Killing field $Z \in \mathfrak{g}$, consider $A=\operatorname{Ker} L_{Z}$ and $B=\operatorname{Im} L_{Z}$, where $L_{Z}: \mathfrak{g} \rightarrow \mathfrak{g}, L_{Z}(X)=[Z, X]$. Then the following conditions are equivalent:

1) $Z$ has constant length on $(M, g)$;

2) $g([Z,[Z, Y]], Z)=0$ on $(M, g)$ for every $Y \in \mathfrak{g}$;

3) $g(Z, Y)=0$ on $(M, g)$ for any $Y \in B$. 
Proof. If $Z$ is of constant length, then for any $X \in \mathfrak{g}$ we have $g([Z, X], Z)=0$ at every point of $M$ by lemma 2 . In particular we may choose $X=[Z, Y]$. Therefore, 1 ) implies 2).

Suppose that $g([Z,[Z, Y]], Z)=0$ for every $Y \in \mathfrak{g}$. If we consider any $\operatorname{Ad}(G)$ invariant inner product $\langle\cdot, \cdot\rangle$ on $\mathfrak{g}$, then the operator $L_{Z}: \mathfrak{g} \rightarrow \mathfrak{g}$ is skew-symmetric. Hence the operator $L_{Z}^{2}$ is symmetric with non-positive eigenvalues. We have $\mathfrak{g}=A \oplus B$ and consider $B=B_{1} \oplus \cdots \oplus B_{k}$, where $B_{i}$ are the eigenvalue spaces for the operator $L_{Z}^{2}$ with the eigenvalues $-\lambda_{i}^{2} \neq 0$. For any $Y \in B_{i}$ we have

$$
0=g([Z,[Z, Y]], Z)=g\left(L_{Z}^{2}(Y), Z\right)=-\lambda_{i}^{2} g(Y, Z) .
$$

Therefore, $g\left(Z, B_{i}\right)=0$ for any $i=1, \ldots, k$, and 2) implies 3$)$.

Now suppose that $g(Z, Y)=0$ for any $Y \in B$. Then $0=Z \cdot g(Y, Z)=-g([Y, Z], Z)$. Moreover, we obviously have $g([Y, Z], Z)=0$ for any $Y \in A$. By lemma 2 we get that $Z$ has constant length.

Note that similar result are known in the literature, see e.g. lemma 2.4 in [3].

Proof of theorem 1. It is easy to see that $\mathfrak{g}_{i}=A_{i} \oplus B_{i}$, where $A_{i}=\operatorname{Ker} L_{Z_{i}} \cap \mathfrak{g}_{i}=$ $\operatorname{Ker} L_{Z} \cap \mathfrak{g}_{i}$ and $B_{i}=\operatorname{Im} L_{Z_{i}}=\operatorname{Im} L_{Z} \cap \mathfrak{g}_{i}$. Note, that the operators $L_{Z}$ and $L_{Z_{0}+Z_{i}}$ are invertible on $B_{i}$.

Since $\mathfrak{g}_{i}$ is simple, then the pair $\left(\mathfrak{g}_{i}, A_{i}\right)$ is effective (this means that the subalgebra $A_{i}$ contains no nontrivial ideal of $\left.\mathfrak{g}_{i}\right)$. By lemma $6, B_{i}+\left[B_{i}, B_{i}\right]$ is an ideal in $\mathfrak{g}_{i}$, hence $B_{i}+\left[B_{i}, B_{i}\right]=\mathfrak{g}_{i}$.

Since the operators $L_{Z}$ invertible on $B_{i}$, we get $g\left(B_{i}, Z\right)=0$ for any $1 \leq i \leq l$ by proposition 2 . Take any $j \neq i, 1 \leq j \leq l$. Then

$$
0=B_{j} \cdot g\left(B_{i}, Z\right)=g\left(B_{i},\left[B_{j}, Z\right]\right),
$$

since $\left[B_{i}, B_{j}\right]=0$. Then we get that $g\left(B_{i}, B_{j}\right)=0$ (we have used that the operator $L_{Z}$ is invertible on $\left.B_{j}\right)$. Further, $0=B_{i} \cdot g\left(B_{i}, B_{j}\right)=g\left(\left[B_{i}, B_{i}\right], B_{j}\right)$. Therefore, $g\left(\mathfrak{g}_{i}, B_{j}\right)=0$, and $0=B_{j} \cdot g\left(\mathfrak{g}_{i}, B_{j}\right)=g\left(\mathfrak{g}_{i},\left[B_{j}, B_{j}\right]\right)$. Hence, $g\left(\mathfrak{g}_{i}, \mathfrak{g}_{j}\right)=0$, that proves the first assertion.

Since $Z$ has constant length, then by proposition 2 , we get $g([Z,[Z, Y]], Z)=0$ for every $Y \in B_{i}$. But in this case $L_{Z}^{2}(Y)=L_{Z_{0}+Z_{i}}^{2}(Y)$ and $g\left(\mathfrak{g}_{i}, Z_{j}\right)=0$ for any $j \neq i$. Therefore, we get $g\left(\left[Z_{0}+Z_{i},\left[Z_{0}+Z_{i}, Y\right]\right], Z_{0}+Z_{i}\right)=0$ for any $Y \in B_{i}$. It is obvious that the same is true for $Y \in A_{i}$. It is clear also that $g\left(\left[Z_{0}+Z_{i},\left[Z_{0}+Z_{i}, Y\right]\right], Z_{0}+Z_{i}\right)=$ 0 for any $Y \in \mathfrak{c}$ and for any $Y \in \mathfrak{g}_{j}, j \neq i$. Therefore, $Z_{0}+Z_{i}$ has constant length by proposition 2 .

Let us prove the last assertion of the theorem. Since $Z_{0}+Z_{i}, 1 \leq i \leq l$, has constant length, then $g\left(\left[Z_{0}+Z_{i},\left[Z_{0}+Z_{i}, Y\right]\right], Z_{0}+Z_{i}\right)=0$ for any $Y \in B_{i}$ by proposition 2. On the other hand, $g\left(\mathfrak{g}_{i}, \mathfrak{g}_{j}\right)=0$ for every $i \neq j, 1 \leq i, j \leq l$. Therefore, $g([Z,[Z, Y]], Z)=g\left(\left[Z_{0}+Z_{i},\left[Z_{0}+Z_{i}, Y\right]\right], Z\right)=0$ for any $Y \in B_{i}$. This equality holds for every $1 \leq i \leq l$. It is easy to see that $g([Z,[Z, Y]], Z)=0$ for any $Y \in \mathfrak{c}$ and for any $Y \in \mathfrak{g}_{j}, j>l$. Therefore, $Z$ has constant length by proposition 2 .

Proof of corollary 1. For $X \in \mathfrak{g}_{i}$ and $Y \in \mathfrak{g}_{j}$, where $i \neq j$, we have $g(X, Y)=0$ by theorem 1 . Then by lemma 3 , we have $\nabla_{X} Y=0$, because $[X, Y]=0$. Moreover, for any $Z \in \mathfrak{g}_{i}$ we get $0=Z \cdot g(X, Y)=g\left(\nabla_{Z} X, Y\right)+g\left(X, \nabla_{Z} Y\right)=g\left(\nabla_{Z} X, Y\right)$. Therefore, $\nabla_{\mathfrak{g}_{i}} \mathfrak{g}_{i} \subset \mathfrak{g}_{i}$ and $\mathfrak{g}_{i}$ is a parallel distribution. Since $i=1, \ldots, k$ is an arbitrary, we get a global splitting by the de Rham theorem (see e.g. theorem 6.1 of chapter IV in [18]) for simply connected $M$. 
The rest of this section is devoted to the proof of theorems 2 and 3 . Let us consider a Killing field $Z \in \mathfrak{g}$ of constant length. Recall that the operators $L_{Z}$ and $L_{Z}^{2}$ are skew-symmetric and symmetric respectively.

We consider $\mathfrak{m}_{0}=\operatorname{Ker} L_{Z}=\{Y \in \mathfrak{g} \mid[Z, Y]=0\}$ and the eigenspace decomposition

$$
\begin{aligned}
\mathfrak{m} & =\mathfrak{m}_{\lambda_{1}} \oplus \mathfrak{m}_{\lambda_{2}} \oplus \cdots \oplus \mathfrak{m}_{\lambda_{s}}=\operatorname{Im} L_{Z}, \\
\mathfrak{m}_{\lambda_{i}} & =\left\{Y \in \mathfrak{g} \mid L_{Z}^{2}(Y)=[Z,[Z, Y]]=-\lambda_{i}^{2} Y\right\} .
\end{aligned}
$$

Since for any $Y \in \mathfrak{m}_{\lambda_{i}}$ we have $L_{Z}^{2}(Y)=[Z,[Z, Y]]=-\lambda_{i}^{2} Y$, then $\lambda_{i}^{2} g(Z, Y)=$ $-g([Z,[Z, Y]], Z)=0$ by proposition 2 . Therefore, we get

Corollary 3. The equality $g(Z, \mathfrak{m})=0$ holds on $(M, g)$.

Proposition 3. Suppose that $\mathfrak{p}$ is an ad $\left(\mathfrak{m}_{0}\right)$-invariant submodule in $\mathfrak{m}$, and $\mathfrak{p}^{\perp}$ is its $\langle\cdot, \cdot\rangle$-orthogonal complement in $\mathfrak{m}$. Then $g\left(\mathfrak{p}, \mathfrak{p}^{\perp}\right)=0$ on $(M, g)$. In particular, $g\left(\mathfrak{m}_{\alpha}, \mathfrak{m}_{\beta}\right)=0$ on $(M, g)$ for $\alpha \neq \beta$.

Proof. By corollary 3 we have $g(Z, \mathfrak{m})=0$. Let us consider any $X \in \mathfrak{p}$ and any $Y \in \mathfrak{p}^{\perp}$. Then $0=Y \cdot g(Z, X)=g([Y, Z], X)+g(Z,[Y, X])$. Note that $\langle[Y, X], W\rangle=$ $\langle Y,[X, W]\rangle=0$ for any $W \in \mathfrak{m}_{0}$, hence $[Y, X] \in \mathfrak{m}$. From this and proposition 2 we get $g(Z,[Y, X])=0$. Therefore, $g([Y, Z], X)=-g\left(L_{Z}(Y), X\right)=0$. Since $\mathfrak{p}$ is an invariant subspace of $L_{Z}$, and $L_{Z}$ is invertible on $\mathfrak{m}$, this proves the proposition.

Now we consider decomposition (8) in our case: $\mathfrak{m}=\mathfrak{p}_{1} \oplus \mathfrak{p}_{2} \oplus \cdots \oplus \mathfrak{p}_{t}$. From proposition 3 we obviously get

Corollary 4. The equality $g\left(\mathfrak{p}_{i}, \mathfrak{p}_{j}\right)=0$ holds on $(M, g)$ for every $i \neq j$.

We will need the following construction: For all $i \geq 1$ and all $X \in \mathfrak{p}_{i}$ we define

$$
h(X)=[X, \sigma(X)]
$$

where $\sigma(X)$ is defined by the equality $(6): \lambda_{i} \sigma(Y)=[Z, Y]$.

Proposition 4. For all $i \geq 1$ and all $X \in \mathfrak{p}_{i}$ we have $h(X) \in \mathfrak{m}_{0}$ and $g(X, \sigma(X))=$ $g(X, h(X))=g(\sigma(X), h(X))=0$ on $(M, g)$. Moreover, for any $j \neq i, j \geq 1$, the equality $g\left(h(U), \mathfrak{p}_{j}\right)=0$ holds on $(M, g)$.

Proof. It is easy to see that $[X, \sigma(X)]^{+}=0$ (see (7)) and $h(X)=[X, \sigma(X)]=$ $[X, \sigma(X)]^{-} \in \mathfrak{m}_{0}$ for every $X \in \mathfrak{p}_{i}$ and $i \geq 1$ by proposition 1 . Further, we have $g(Z, X)=g(Z, \sigma(X))=0$ by corollary 3 . Hence

$0=X \cdot g(Z, X)=g([X, Z], X)=-\alpha g(\sigma(X), X)$, where $\alpha$ is taken from $\mathfrak{p}_{i} \subset \mathfrak{m}_{\alpha}$,

$0=X \cdot g(X, \sigma(X))=g(X, h(X))$, and $0=\sigma(X) \cdot g(X, \sigma(X))=-g(h(X), \sigma(X))$.

Now, we prove the last assertion. Take any $Y \in \mathfrak{p}_{j} \subset \mathfrak{m}_{\beta}$. By corollary 4 , we have $g(\sigma(X), Y)=g(\sigma(X), \sigma(Y))=0$, therefore,

$$
\begin{aligned}
& 0=X \cdot g(\sigma(X), Y)=g(h(X), Y)+g(\sigma(X),[X, Y]), \\
& 0=\sigma(X) \cdot g(\sigma(X), \sigma(Y))=g(\sigma(X),[\sigma(X), \sigma(Y)]) .
\end{aligned}
$$

From this we get

$$
-g(h(X), Y)=g(\sigma(X),[X, Y] \pm[\sigma(X), \sigma(Y)])=g\left(\sigma(X),[X, Y]^{ \pm}\right) .
$$

By proposition 1 , we have $[X, Y]^{+} \in \mathfrak{m}_{\alpha+\beta}$ and $[X, Y]^{-} \in \mathfrak{m}_{|\alpha-\beta|}$. Since $\alpha+\beta>\alpha$, we obtain $g\left(\sigma(X),[X, Y]^{+}\right)=0$ by proposition 3. Therefore, $g(h(X), Y)=0$. 
Proposition 5. Suppose that $i \geq 1$ and $W \in \mathfrak{m}_{0}$ are such that $\langle W, h(X)\rangle=0$ for all $X \in \mathfrak{p}_{i}$. Then $\left[W, \mathfrak{p}_{i}\right]=0$.

Proof. Consider the operators $P, Q: \mathfrak{p}_{i} \rightarrow \mathfrak{p}_{i}$, such that $P(X)=[W, X]$ and $Q(X)=[Z, X]$. These operators are skew-symmetric with respect to $\langle\cdot, \cdot\rangle$ and commute one with other (since $[Z, W]=0$ ). Moreover, $Q^{2}=-\lambda_{k}^{2} \mathrm{Id}$, where $\mathfrak{p}_{i} \subset \mathfrak{m}_{\lambda_{k}}$. Therefore, the operator $R:=P Q(=Q P)$ is symmetric and $R^{2}=P Q^{2} P=-\lambda_{k}^{2} P^{2}$. Now, for any $U \in \mathfrak{p}_{i}$ we get

$$
\begin{array}{r}
\left.0=-\lambda_{k}\langle W, h(U)\rangle=-\langle W,[U,[Z, U]]\rangle=\langle[U, W],[Z, U]]\right\rangle= \\
-\langle[W, U],[Z, U]]\rangle=\langle[Z,[W, U]], U\rangle=\langle R(U), U\rangle .
\end{array}
$$

Therefore, $R=0$ and $P=0$, i.e. $\left[W, \mathfrak{p}_{i}\right]=0$.

Proposition 6. Let $Z=Z_{0}+Z_{1} \in \mathfrak{g}$ be a Killing field of constant length on $(M, g)$, where $Z_{0} \in \mathfrak{c}, Z_{1} \in \mathfrak{g}_{1}, Z_{1} \neq 0$, and let $\mathfrak{k}$ be the centralizer of $Z$ (and $Z_{1}$ ) in $\mathfrak{g}_{1}$. Then one of the following statements holds:

1) The equality $g(\mathfrak{k}, \mathfrak{m})=0$ is fulfilled on $(M, g)$;

2) The Lie algebra pair $\left(\mathfrak{g}_{1}, \mathfrak{k}\right)$ is irreducible Hermitian symmetric and the center of $\mathfrak{k}$ is a one-dimensional Lie algebra spanned by the vector $Z_{1}$.

Proof. We apply all above constructions of this section to prove this key proposition. Note that

$$
\mathfrak{m}=\mathfrak{p}_{1} \oplus \cdots \oplus \mathfrak{p}_{t} \subset \mathfrak{g}_{1} \quad \text { and } \quad \mathfrak{m}_{0}=\mathfrak{c} \oplus \mathfrak{k} \oplus \mathfrak{g}_{2} \oplus \mathfrak{g}_{3} \oplus \cdots \oplus \mathfrak{g}_{k}
$$

in our case.

First, let us suppose that $\left[\mathfrak{p}_{i}, \mathfrak{p}_{j}\right]=0$ for all $i \neq j, 1 \leq i, j \leq s$. As we have noted, the first decomposition in (13) and the decomposition $\mathfrak{g}_{1}=\mathfrak{k} \oplus \mathfrak{m}$ are orthogonal with respect to $\langle\cdot, \cdot\rangle$. Now, by lemma 7 we get that every $\mathfrak{p}_{i}+\left[\mathfrak{p}_{i}, \mathfrak{p}_{i}\right], 1 \leq i \leq s$, is an ideal in $\mathfrak{g}_{1}$. Since $\mathfrak{g}_{1}$ is a simple Lie algebra, we get that $s=1$, and $\mathfrak{p}_{1}=\mathfrak{m}_{\lambda_{1}}$.

For $X, Y \in \mathfrak{p}_{1}$, proposition 1 implies $[X, Y]^{+}=1 / 2([U, V]-[\sigma(U), \sigma(V)]) \in \mathfrak{m}_{2 \lambda_{1}}$, hence $[X, Y]^{+}=0$ (recall that $s=1$ and $\lambda_{1}<2 \lambda_{1}$ ) and $[X, Y]=[X, Y]^{-}=$ $1 / 2([X, Y]+[\sigma(X), \sigma(Y)]) \in \mathfrak{m}_{0}$. This means that $\left[\mathfrak{p}_{1}, \mathfrak{p}_{1}\right] \subset \mathfrak{k}$. Therefore, the Lie algebra pair $\left(\mathfrak{g}_{1}, \mathfrak{k}\right)$ is irreducible symmetric. But $\mathfrak{k}$ is the centralizer of the vector $Z_{1}$ in $\mathfrak{g}_{1}$, therefore, it has non-zero center. Consequently, $\left(\mathfrak{g}_{1}, \mathfrak{k}\right)$ is an Hermitian irreducible symmetric pair (see e.g. proposition 8.7.12 in [32]).

In the rest of the proof we will assume that there are indices $i \neq j, 1 \leq i, j \leq s$, such that $\left[\mathfrak{p}_{i}, \mathfrak{p}_{j}\right] \neq 0$.

Let $S$ be a linear span of all vectors $h(U)$ (see (12)), where $U \in \mathfrak{p}_{i}, i \geq 1$. In our case $\mathfrak{p}_{i} \subset \mathfrak{g}_{1}$, hence $h(U) \in \mathfrak{g}_{1}$ and $S \subset \mathfrak{g}_{1}$. We prove that the equality $g\left(S,\left[\mathfrak{p}_{i}, \mathfrak{p}_{j}\right]\right)=0$ holds on $(M, g)$ for all $i, j \geq 1, i \neq j$.

Take any $h(U)$, where $U \in \mathfrak{p}_{k}, k \geq 1$. Without loss of generality we may suppose that $k \neq j$. Then $g\left(h(U), \mathfrak{p}_{j}\right)=0$ by proposition 4 . Now, for every $X \in \mathfrak{p}_{i}$ and $Y \in \mathfrak{p}_{j}$ we have $0=X \cdot g(h(U), Y)=g([X, h(U)], Y)+g(h(U),[X, Y])$. Since $[h(U), X] \in$ $\left[\mathfrak{m}_{0}, \mathfrak{p}_{i}\right] \subset \mathfrak{p}_{i}$ we have $g([X, h(U)], Y)=0$ by corollary 4 . Hence, $g(h(U),[X, Y])=0$ that implies $g\left(h(U),\left[\mathfrak{p}_{i}, \mathfrak{p}_{j}\right]\right)=0$ and $g\left(S,\left[\mathfrak{p}_{i}, \mathfrak{p}_{j}\right]\right)=0$.

Let $S^{\perp}$ be the orthogonal complement to the linear space $S$ in $\mathfrak{m}_{0}$ with respect to $\langle\cdot, \cdot\rangle$. By proposition 5 we have $\left[W, \mathfrak{p}_{i}\right]=0$ for all $W \in S^{\perp}$ and all $i$, i.e. $\left[S^{\perp}, \mathfrak{m}\right]=0$. 
Let $q_{1}$ be a maximal (by inclusion) linear subspace in $\mathfrak{m}_{0}$ with the property $\left[q_{1}, \mathfrak{m}\right]=0$. We get that $q_{1}$ is an ideal in $\mathfrak{g}$ by lemma 6 .

Note also that $\mathfrak{c} \oplus \mathfrak{g}_{2} \oplus \mathfrak{g}_{3} \oplus \cdots \oplus \mathfrak{g}_{k} \subset S^{\perp} \subset q_{1}$. Since the Lie algebra $\mathfrak{g}_{1}$ is simple we get $q_{1}=S^{\perp}=\mathfrak{c} \oplus \mathfrak{g}_{2} \oplus \mathfrak{g}_{3} \oplus \cdots \oplus \mathfrak{g}_{k}$, therefore, $S=\mathfrak{k}$. Consequently, we get that $g\left(\mathfrak{k},\left[\mathfrak{p}_{i}, \mathfrak{p}_{j}\right]\right)=0$ for every $i \neq j, 1 \leq i, j \leq t$.

Since $\left[\mathfrak{m}_{0},\left[\mathfrak{p}_{i}, \mathfrak{p}_{j}\right]\right] \subset\left[\left[\mathfrak{m}_{0}, \mathfrak{p}_{i}\right], \mathfrak{p}_{j}\right]+\left[\mathfrak{p}_{i},\left[\mathfrak{m}_{0}, \mathfrak{p}_{j}\right]\right] \subset\left[\mathfrak{p}_{i}, \mathfrak{p}_{j}\right]$, then any subspace of the type $\left[\mathfrak{p}_{i}, \mathfrak{p}_{j}\right] \subset \mathfrak{m}$ is ad $\left(\mathfrak{m}_{0}\right)$-invariant.

Let $r_{1}$ be a maximal (by inclusion) ad $\left(\mathfrak{m}_{0}\right)$-invariant linear subspace in $\mathfrak{m}$ with the property $g\left(\mathfrak{k}, r_{1}\right)=0$. Clearly, $\left[\mathfrak{p}_{i}, \mathfrak{p}_{j}\right] \subset r_{1}$ for any $i \neq j$. We are going to prove that $r_{1}=\mathfrak{m}$.

Denote by $r_{2}$ the $\langle\cdot, \cdot\rangle$-orthogonal complement to $r_{1}$ in $\mathfrak{m}$. Since both $r_{1}$ and $r_{2}$ are spanned by some modules $\mathfrak{p}_{j}$, we get that $\left[r_{1}, r_{2}\right] \subset r_{1}$, because $\left[\mathfrak{p}_{i}, \mathfrak{p}_{j}\right] \subset r_{1}$ for any $i \neq j$. Since $\left\langle\left[r_{2}, r_{2}\right], r_{1}\right\rangle=-\left\langle r_{2},\left[r_{2}, r_{1}\right]\right\rangle=0$, then $\mathfrak{k} \oplus r_{2}$ is a Lie subalgebra of $\mathfrak{g}_{1}$.

Let $S_{1}$ be a linear span of all vectors $h(U)$ (see (12)), where $U \in \mathfrak{p}_{i}$ and $\mathfrak{p}_{i} \subset r_{1}$. We know that $h(U) \in \mathfrak{k}$.

Let $S_{1}^{\perp}$ be an orthogonal complement to the linear space $S_{1}$ in $\mathfrak{k}$ with respect to $\langle\cdot, \cdot\rangle$. By proposition 5 we have $\left[W, \mathfrak{p}_{i}\right]=0$ for all $W \in S_{1}^{\perp}$ and $r_{1} \subset \mathfrak{p}_{1}$, i.e. $\left[S_{1}^{\perp}, r_{1}\right]=0$. Let $q_{2}$ be a maximal (by inclusion) linear subspace in $\mathfrak{k} \oplus r_{2}$ with the property $\left[q_{2}, r_{1}\right]=0$. Therefore, $q_{2}$ is an ideal in $\mathfrak{g}_{1}$ by lemma 6 . Since $\mathfrak{g}_{1}$ is a simple Lie algebra, we get $q_{2}=0$. Since $S_{1}^{\perp} \subset q_{2}$, we get $S_{1}=\mathfrak{k}$.

For all $i \geq 1$ and all $U \in \mathfrak{p}_{i}$ with $\mathfrak{p}_{i} \subset r_{1}$, and for all $\mathfrak{p}_{j} \subset r_{2}$, we have the equality $g\left(\mathfrak{p}_{j}, h(U)\right)=0$ on $(M, g)$ by proposition 4 . Therefore, $g\left(\mathfrak{p}_{j}, S_{1}\right)=g\left(\mathfrak{p}_{j}, \mathfrak{k}\right)=0$ for any $\mathfrak{p}_{j} \subset r_{2}$. Hence, $g\left(r_{2}, \mathfrak{k}\right)=0$ and $r_{2}=0$ by the definition of $r_{1}$. Consequently, we get the equality $g(\mathfrak{k}, \mathfrak{m})=0$ on $(M, g)$.

Proposition 7. If the case 1) of proposition 6 holds, then the every Killing field $Y \in \mathfrak{g}_{1}$ (in particular, $Z_{1}$ ) has constant length on $(M, g)$.

Proof. According to the case 1) of proposition 6 , we get that $g(\mathfrak{k}, \mathfrak{m})=0$ at every point of $M$, where $\mathfrak{k}$ is the centralizer of $Z_{1}$ in $\mathfrak{g}_{1}$ and $\mathfrak{m}$ is the ad( $\left.\mathfrak{k}\right)$-invariant complement to $\mathfrak{k}$ in $\mathfrak{g}_{1}$.

Let us consider any Cartan subalgebra $\mathfrak{t}$ in $\mathfrak{g}_{1}$ such that $Z_{1} \in \mathfrak{t}$. First, prove that every $X \in \mathfrak{t}$ has constant length on $(M, g)$. By proposition 2, it suffices to prove that $g\left(\mathfrak{t}, \mathfrak{v}_{\alpha}\right)=0$ for all $\alpha \in \Delta^{+}$in the decomposition (9), where $\mathfrak{g}:=\mathfrak{g}_{1}$. Indeed, take any $X \in \mathfrak{t}$. Note that $g([X,[X, Y]], X)=0$ for all $Y \in \mathfrak{t}$, because $[X, Y]=0$. Moreover, $g([X,[X, Y]], X)=0$ is easily satisfied if $Y$ is from other factors of $\mathfrak{g}$. If $Y \in \mathfrak{v}_{\alpha}$ for $\alpha \in \Delta^{+}$, then $g([X,[X, Y]], X)=-\left\langle X, \alpha_{i}\right\rangle^{2} g(X, Y)$ by (10). Therefore, if $g\left(X, \mathfrak{v}_{\alpha}\right)=0$ for all $\alpha \in \Delta^{+}$, then $g([X,[X, Y]], X)=0$ for every $Y \in \mathfrak{g}$. By proposition 2 it implies that $X$ has constant length on $(M, g)$.

We have the equality $g(\mathfrak{t}, \mathfrak{m})=0$. Let us consider the orbit $\left\{Z_{1}=U_{1}, U_{2}, \ldots, U_{l}\right\}$ of $Z_{1}$ in $\mathfrak{t}$ under the action of the Weyl group $W$, see section 4 . By properties of $W$, for any $U_{i}$ there is an inner automorphism $\psi_{i}$ of $\mathfrak{g}_{1}$ such that $\mathfrak{t}$ is stable under $\psi_{i}$ and $U_{i}=\psi_{i}\left(Z_{1}\right)$. Note that we may consider $\psi_{i}$ as an automorphism of $\mathfrak{g}=$ $\mathfrak{c} \oplus \mathfrak{g}_{1} \oplus \cdots \oplus \mathfrak{g}_{k}$ (it acts identically on all other summands). By lemma 5 , the Killing field $Z_{0}+U_{i}=Z_{0}+\psi_{i}\left(Z_{1}\right)=\psi_{i}\left(Z_{0}+Z_{1}\right)$ also has constant length. Moreover, $g\left(\psi_{i}(\mathfrak{t}), \psi_{i}(\mathfrak{m})\right)=g\left(\mathfrak{t}, \psi_{i}(\mathfrak{m})\right)=0$ on $(M, g)$ by the same lemma. Now, it suffices to prove that for every $\alpha \in \Delta^{+}$, there is $i$ such that $\mathfrak{v}_{\alpha} \subset \psi_{i}(\mathfrak{m})$. The latter is equivalent 
to the following: $\psi_{i}\left(Z_{1}\right)=U_{i}$ is not orthogonal to the root $\alpha$ with respect to $\langle\cdot, \cdot\rangle$, because $\psi_{i}(\mathfrak{k})$ is the centralizer of $U_{i}=\psi_{i}\left(Z_{1}\right)$ in $\mathfrak{g}_{1}$ and $\psi_{i}(\mathfrak{m})$ consists of all root spaces with $\left\langle\alpha, U_{i}\right\rangle \neq 0$.

Suppose that there is no suitable $\psi_{i}\left(Z_{1}\right)=U_{i}$, i.e. $\left\langle\alpha, U_{i}\right\rangle=0$ for all $i$. But $W$ acts irreducibly on $\mathfrak{t}$, therefore $\operatorname{Lin}\left(Z_{1}=U_{1}, U_{2}, \ldots, U_{l}\right)=\mathfrak{t}$. This contradiction proves $g\left(\mathfrak{t}, \mathfrak{v}_{\alpha}\right)=0$, therefore every $X \in \mathfrak{t}$ has constant length on $(M, g)$.

Now, for an arbitrary $X \in \mathfrak{g}_{1}$, there is an inner automorphism $\eta$ of $\mathfrak{g}_{1}$ such that $\eta(X) \in \mathfrak{t}$, see section 4 . Then using lemma 5 again, we obtain that $X$ has constant length on $(M, g)$.

We will finish the proof of theorem 2 in the next section. But now we are going to prove theorem 3 .

Lemma 9. Let $G$ be compact Lie group (with the Lie algebra $\mathfrak{g}$ ) supplied with a left-invariant Riemannian metric $\mu$. If every $X \in \mathfrak{g}$ has constant length on the Riemannian manifold $(G, \mu)$, then $\mu$ is a bi-invariant metric.

Proof. Since for every Killing field $X, Y \in \mathfrak{g}$ we have

$$
2 \mu(X, Y)=\mu(X+Y, X+Y)-\mu(X, X)-\mu(Y, Y)=\text { const, }
$$

then for any $a \in G$

$$
\mu_{e}(\operatorname{Ad}(a)(X), \operatorname{Ad}(a)(Y))=\mu_{a}(\operatorname{Ad}(a)(X), \operatorname{Ad}(a)(Y))=\mu_{e}(X, Y)
$$

by lemma 5 , where $e$ is the unit in $G$. Therefore, $\mu$ is a bi-invariant metric on $G$.

Proof of theorem 3. Since $Z$ is regular, then $k=l$ in theorem 1 and every $Z_{i}$ is regular in $\mathfrak{g}_{i}$. By theorem 1 we have $g\left(\mathfrak{g}_{i}, \mathfrak{g}_{j}\right)=0$ for every $i \neq j, 1 \leq i, j \leq k$, and all $Z_{0}+Z_{i}$ have constant length on $(M, g)$. Since $Z_{i}$ is regular in $\mathfrak{g}_{i}$, we get the case 1) in proposition 6 and, therefore, all $X \in \mathfrak{g}_{i}$ has constant length on $(M, g)$ by proposition 7 (here we have considered $\mathfrak{g}_{i}$ instead of $\mathfrak{g}_{1}$ ). Therefore, every $X \in \mathfrak{g}_{\mathfrak{s}}$, where $\mathfrak{g}_{\mathfrak{s}}=\mathfrak{g}_{1} \oplus \cdots \oplus \mathfrak{g}_{k}$ is a semisimple part of $\mathfrak{g}$, also has constant length. In particular, $Z_{\mathfrak{s}}:=Z-Z_{0}$ has constant length. By proposition 2 we get $g(Z, Y)=$ $g\left(Z_{\mathfrak{s}}, Y\right)=0$ for every $Y \in \mathfrak{m}$, hence, $g\left(Z_{0}, Y\right)=0$ for the same $Y$. Now, for any $X \in \mathfrak{m}$ we have $0=X \cdot g\left(Z_{0}, Y\right)=g\left(Z_{0},[X, Y]\right)$. Note that $\mathfrak{m}+[\mathfrak{m}, \mathfrak{m}]$ is an ideal in $\mathfrak{g}_{\mathfrak{s}}$ by lemma 6 , hence, $\mathfrak{m}+[\mathfrak{m}, \mathfrak{m}]=\mathfrak{g}_{\mathfrak{s}}$ and $g\left(Z_{0}, \mathfrak{g}_{\mathfrak{s}}\right)=0$.

Now, suppose that $M$ is simply connected. In this case semisimple part of the isometry group $G$ acts transitively on $(M, g)$ (see e.g. proposition 9 on p. 94 in [24]). This mean that the Lie algebra $\mathfrak{g}_{\mathfrak{s}}$ generates a tangent space to $M$ at every point $x \in M$. Since $g\left(Z_{0}, \mathfrak{g}_{\mathfrak{s}}\right)=0$ on $M$, we get $Z_{0}=0$.

Further, $(M, g)$ is a direct metric product of Riemannian manifold $\left(M_{i}, g_{i}\right)$, that are corresponded to the distributions $\mathfrak{g}_{i}$, by corollary 1 . Therefore, $G_{i}$ is an isometry group of $\left(M_{i}, g_{i}\right)$. Since all $X \in \mathfrak{g}_{i}$ have constant length, the isotropy group (of any point of $\left.\left(M_{i}, g_{i}\right)\right)$ is discrete. Since $M_{i}$ is simply connected, $G_{i}$ acts simply transitively on $\left(M_{i}, g_{i}\right)$, hence $\left(M_{i}, g_{i}\right)$ is isometric to $G_{i}$ supplied with some left-invariant Riemannian metric $\mu_{i}$. Since all $X \in \mathfrak{g}_{i}$ have constant length on $\left(G_{i}, \mu_{i}\right)$, then $\mu_{i}$ is bi-invariant by lemma 9 .

\section{More detailed study of the Hermitian CASE}

In this section we study in more details the case 2 ) in proposition 6 , which we call Hermitian. In this case, $\mathfrak{m}$ is $\operatorname{ad}(\mathfrak{k})$-irreducible, in particular $\left.L_{Z}^{2}\right|_{\mathfrak{m}}=-C \cdot \operatorname{Id}$, where $C$ 
is a positive constant. Multiply $Z$ by a suitable constant we may suppose that $C=1$. Using the root space decomposition (9) for $\mathfrak{g}_{1}$ we get

$$
\mathfrak{m}=\bigoplus_{\alpha \in I_{1}(Z)} \mathfrak{v}_{\alpha}, \quad \mathfrak{k}=\mathfrak{t} \oplus \bigoplus_{\alpha \in I_{2}(Z)} \mathfrak{v}_{\alpha}
$$

where

$$
I_{1}(Z)=\left\{\alpha \in \Delta^{+} \mid\langle Z, \alpha\rangle \neq 0\right\}, \quad I_{2}(Z)=\left\{\alpha \in \Delta^{+} \mid\langle Z, \alpha\rangle=0\right\} .
$$

Without loss of generality we may assume that $Z$ is in the Cartan chamber $C\left(\Delta^{+}\right)$ (see (11)), hence $\langle Z, \alpha\rangle>0$ for all $\alpha \in I_{1}(Z)$. By (10) we have

$$
\left[Z, U_{\alpha}\right]=\langle Z, \alpha\rangle V_{\alpha}, \quad\left[Z, V_{\alpha}\right]=-\langle Z, \alpha\rangle U_{\alpha},\left.\quad L_{Z}^{2}\right|_{\mathfrak{v}_{\alpha}}=-\langle Z, \alpha\rangle^{2} \cdot \mathrm{Id}
$$

for all $\alpha \in I_{1}(Z)$. Then, $\langle Z, \alpha\rangle=1$ for all $\alpha \in I_{1}(Z)$ by our assumptions. In particular, $\left.L_{Z}\right|_{\mathfrak{m}}=\sigma$, see (6). By $W=W(\mathfrak{t})$ we denote the corresponding Weyl group of $\mathfrak{g}_{1}$.

Remark 1. We can explain the connection of the Hermitian case with Hermitian symmetric spaces as follows. Let $\alpha_{\max }=\sum_{i} a_{i} \pi_{i}$ be a maximal root for $\Delta^{+}$. Then $\left\langle Z, \alpha_{\max }\right\rangle=\sum_{i} a_{i}\left\langle Z, \pi_{i}\right\rangle$, where all $a_{i}$ are integer with $a_{i} \geq 1$. Note that $\left\langle Z, \pi_{i}\right\rangle=0$ for $\pi_{i} \in I_{2}(Z)$ and $\left\langle Z, \pi_{i}\right\rangle=1$ for $\pi_{i} \in I_{1}(Z)$. There is at least one $\pi_{j}$ in $I_{1}(Z)$, hence $\left\langle Z, \alpha_{\max }\right\rangle=1$, and such $\pi_{j}$ should be unique, moreover, $a_{j}=1$. Therefore, $\pi_{j}$ is a non-compact root and $\pi_{i} \in I_{2}(Z)$ for all $i \neq j$. Every non-compact root determines a Hermitian symmetric space as it was shown in [31].

Lemma 10. In the above notations, for any $w \in W$ the Killing field $Z_{0}+w\left(Z_{1}\right)$ has constant length on $(M, g)$.

Proof. By properties of $W$ (see section 4), there is an inner automorphism $\psi$ of $\mathfrak{g}_{1}$ such that $\mathfrak{t}$ is stable under $\psi$ and $w\left(Z_{1}\right)=\psi\left(Z_{1}\right)$. We may consider $\psi$ as an automorphism of $\mathfrak{g}=\mathfrak{c} \oplus \mathfrak{g}_{1} \oplus \cdots \oplus \mathfrak{g}_{k}$ (it acts identically on all other summands). By lemma 5 , the Killing field $Z_{0}+w\left(Z_{1}\right)=Z_{0}+\psi\left(Z_{1}\right)=\psi_{i}\left(Z_{0}+Z_{1}\right)$ also has constant length.

Proposition 8. In the notation as above, for any $\alpha \in I_{1}(Z)$, the following equalities hold:

$$
\begin{gathered}
g\left(Z, \mathfrak{v}_{\alpha}\right)=0, \quad g\left(U_{\alpha}, U_{\alpha}\right)=g\left(V_{\alpha}, V_{\alpha}\right)=g(\alpha, Z)=\frac{1}{\langle\alpha, \alpha\rangle} g(\alpha, \alpha), \\
g\left(U_{\alpha}, V_{\alpha}\right)=g\left(\alpha, U_{\alpha}\right)=g\left(\alpha, V_{\alpha}\right)=0, \quad g(\alpha, \alpha)=\langle\alpha, \alpha\rangle g(\alpha, Z) .
\end{gathered}
$$

Proof. The equality $g\left(Z, \mathfrak{v}_{\alpha}\right)=0$ follows from corollary 3. Substituting $X:=Z$, $Z:=V_{\alpha}$ and $Y:=U_{\alpha}$ in the equality $g([Z,[Y, X]], X)+g([Y, X],[Z, X])=0$ (see $(4)$ in lemma 2) and using $g\left(Z, \mathfrak{v}_{\alpha}\right)=0$ and (10), we get

$$
0=g\left(\left[V_{\alpha},\left[U_{\alpha}, Z\right]\right], Z\right)+g\left(\left[\left[U_{\alpha}, Z\right],\left[V_{\alpha}, Z\right]\right)=-\langle Z, \alpha\rangle^{2} g\left(U_{\alpha}, V_{\alpha}\right)=-g\left(U_{\alpha}, V_{\alpha}\right) .\right.
$$

Further,

$$
\begin{gathered}
0=U_{\alpha} \cdot g\left(U_{\alpha}, V_{\alpha}\right)=g\left(U_{\alpha},\left[U_{\alpha}, V_{\alpha}\right]\right)=g\left(U_{\alpha}, \alpha\right), \\
0=V_{\alpha} \cdot g\left(U_{\alpha}, V_{\alpha}\right)=g\left(\left[V_{\alpha}, U_{\alpha}\right], V_{\alpha}\right)=-g\left(\alpha, V_{\alpha}\right) .
\end{gathered}
$$

Since $g\left(U_{\alpha}, Z\right)=0$, then

$$
0=V_{\alpha} \cdot g\left(U_{\alpha}, Z\right)=-g(\alpha, Z)+g\left(U_{\alpha},\langle Z, \alpha\rangle U_{\alpha}\right)=g\left(U_{\alpha}, U_{\alpha}\right)-g(\alpha, Z) \text {. }
$$


Analogously,

$$
0=U_{\alpha} \cdot g\left(V_{\alpha}, Z\right)=g(\alpha, Z)+g\left(V_{\alpha},-\langle Z, \alpha\rangle V_{\alpha}\right)=-g\left(V_{\alpha}, V_{\alpha}\right)+g(\alpha, Z) .
$$

Further, since $g\left(U_{\alpha}, \alpha\right)=g\left(V_{\alpha}, \alpha\right)=0$, we get

$$
\begin{aligned}
& 0=U_{\alpha} \cdot g\left(V_{\alpha}, \alpha\right)=g\left(\left[U_{\alpha}, V_{\alpha}\right], \alpha\right)+g\left(V_{\alpha},\left[U_{\alpha}, \alpha\right]\right)=g(\alpha, \alpha)-\langle\alpha, \alpha\rangle g\left(V_{\alpha}, V_{\alpha}\right), \\
& 0=V_{\alpha} \cdot g\left(U_{\alpha}, \alpha\right)=g\left(\left[V_{\alpha}, U_{\alpha}\right], \alpha\right)+g\left(U_{\alpha},\left[V_{\alpha}, \alpha\right]\right)=\langle\alpha, \alpha\rangle g\left(U_{\alpha}, U_{\alpha}\right)-g(\alpha, \alpha) .
\end{aligned}
$$

The obtained equalities prove the proposition.

Lemma 11. For every $\alpha, \beta \in I_{1}(Z)$ the condition $g\left(\mathfrak{v}_{\alpha}, \mathfrak{v}_{\beta}\right)=0$ is equivalent to the condition $g\left(\left[\mathfrak{v}_{\alpha}, \mathfrak{v}_{\beta}\right], Z\right)=0$. In particular, $g\left(\mathfrak{v}_{\alpha}, \mathfrak{v}_{\beta}\right)=0$ follows from $\left[\mathfrak{v}_{\alpha}, \mathfrak{v}_{\beta}\right] \subset \mathfrak{m}$.

Proof. By proposition 8 we have $g\left(\mathfrak{v}_{\alpha}, Z\right)=g\left(\mathfrak{v}_{\beta}, Z\right)=0$. Further, for any $Y \in \mathfrak{v}_{\alpha}$ and $X \in \mathfrak{v}_{\beta}$ we have

$$
0=X \cdot g(Y, Z)=g([X, Y], Z)+g(Y,[X, Z])=g([X, Y], Z)-g\left(Y, L_{Z}(X)\right),
$$

Since $L_{Z}$ is bijective on $\mathfrak{v}_{\beta}$, this proves the first assertion of the lemma. The second assertion follows from the first one and corollary 3.

Proposition 9. For $\alpha, \beta \in I_{1}(Z)$, the equality $g\left(\alpha, \mathfrak{v}_{\beta}\right)=0$ holds under each of the following conditions:

$$
\text { 1) } \alpha=\beta ; \quad \text { 2) }\langle\alpha, \beta\rangle=0 ; \quad \text { 3) } g\left(\mathfrak{v}_{\alpha}, \mathfrak{v}_{\beta}\right)=0 .
$$

If $X \in \mathfrak{t}$ is such that $g(X, \alpha)=0$, then $g\left(X, \mathfrak{v}_{\alpha}\right)=0$.

Proof. If $\alpha=\beta$, then $g\left(\mathfrak{v}_{\alpha}, \beta\right)=0$ by proposition 8 . Further, suppose that $\langle\alpha, \beta\rangle=0$, which implies $\left[\alpha, \mathfrak{v}_{\beta}\right]=0$. Since $g(\alpha, \alpha)=\langle\alpha, \alpha\rangle g(\alpha, Z)$ by proposition 8 , we get

$$
0=2 g([X, \alpha], \alpha)=X \cdot g(\alpha, \alpha)=\langle\alpha, \alpha\rangle X \cdot g(\alpha, Z)=\langle\alpha, \alpha\rangle g(\alpha,[X, Z])
$$

for any $X \in \mathfrak{v}_{\beta}$, where $\langle\alpha, \beta\rangle=0$ (note that $\left[\alpha, \mathfrak{v}_{\beta}\right]=0$ for $\langle\alpha, \beta\rangle=0$ ). Since $\left[Z, \mathfrak{v}_{\beta}\right]=\mathfrak{v}_{\beta}$, we get $g\left(\alpha, \mathfrak{v}_{\beta}\right)=0$.

Now, suppose that $g\left(\mathfrak{v}_{\alpha}, \mathfrak{v}_{\beta}\right)=0$. If $\langle\alpha, \beta\rangle=0$ then we can apply 2). Consider the case $\langle\alpha, \beta\rangle \neq 0$.

By proposition 8 we have $g\left(U_{\alpha}, U_{\alpha}\right)=g\left(V_{\alpha}, V_{\alpha}\right)=\frac{1}{\langle\alpha, \alpha\rangle} g(\alpha, \alpha)$. From the equality $g\left(\mathfrak{v}_{\alpha}, \mathfrak{v}_{\beta}\right)=0$ we get

$$
\begin{aligned}
& 0=-2 U_{\alpha} \cdot g\left(U_{\alpha}, U_{\beta}\right)=2 g\left(U_{\alpha},\left[U_{\beta}, U_{\alpha}\right]\right)=U_{\beta} \cdot g\left(U_{\alpha}, U_{\alpha}\right), \\
& 0=-2 V_{\alpha} \cdot g\left(V_{\alpha}, V_{\beta}\right)=2 g\left(V_{\alpha},\left[V_{\beta}, V_{\alpha}\right]\right)=V_{\beta} \cdot g\left(V_{\alpha}, V_{\alpha}\right) .
\end{aligned}
$$

Therefore,

$$
\begin{aligned}
& 0=U_{\beta} \cdot g\left(U_{\alpha}, U_{\alpha}\right)=\frac{1}{\langle\alpha, \alpha\rangle}\left(U_{\beta} \cdot g(\alpha, \alpha)\right)=\frac{2}{\langle\alpha, \alpha\rangle} g\left(\left[U_{\beta}, \alpha\right], \alpha\right)=-\frac{2\langle\alpha, \beta\rangle}{\langle\alpha, \alpha\rangle} g\left(V_{\beta}, \alpha\right), \\
& 0=V_{\beta} \cdot g\left(V_{\alpha}, V_{\alpha}\right)=\frac{1}{\langle\alpha, \alpha\rangle}\left(V_{\beta} \cdot g(\alpha, \alpha)\right)=\frac{2}{\langle\alpha, \alpha\rangle} g\left(\left[V_{\beta}, \alpha\right], \alpha\right)=\frac{2\langle\alpha, \beta\rangle}{\langle\alpha, \alpha\rangle} g\left(U_{\beta}, \alpha\right) .
\end{aligned}
$$

Hence, $g\left(\alpha, \mathfrak{v}_{\beta}\right)=0$ in this case too.

Finally, if $g(X, \alpha)=0$ for $X \in \mathfrak{t}$, then for any $Y \in \mathfrak{v}_{\alpha}$ we get $0=Y \cdot g(X, \alpha)=$ $g([Y, X], \alpha)+g(X,[Y, \alpha])$. Since $[Y, X] \in \mathfrak{v}_{\alpha}$, we get $g([Y, X], \alpha) \subset g\left(\mathfrak{v}_{\alpha}, \alpha\right)=0$. Hence, $g(X,[Y, \alpha])=0$. Since $Y$ is an arbitrary in $\mathfrak{v}_{\alpha}$, then $g\left(X, \mathfrak{v}_{\alpha}\right)=0$.

Remark 2. Note that $\left[\mathfrak{v}_{\alpha}, \mathfrak{v}_{\beta}\right]=0$ implies $\langle\alpha, \beta\rangle=0$. Such roots $\alpha$ and $\beta$ are called strongly orthogonal. 
Lemma 12. If $g\left(\mathfrak{v}_{\alpha}, \beta\right)=0$ for some $\alpha, \beta \in I_{1}(Z)$, then

$$
\langle\alpha, \beta\rangle g\left(U_{\alpha}, U_{\alpha}\right)=\langle\alpha, \beta\rangle g\left(V_{\alpha}, V_{\alpha}\right)=g(\alpha, \beta) .
$$

In particular, if $\alpha, \beta \in I_{1}(Z)$ are such that $\langle\alpha, \beta\rangle=0$, then $g(\alpha, \beta)=0$.

Proof. By direct calculations we get

$$
0=U_{\alpha} \cdot g\left(V_{\alpha}, \beta\right)=g\left(\left[U_{\alpha}, V_{\alpha}\right], \beta\right)+g\left(V_{\alpha},\left[U_{\alpha}, \beta\right]\right)=g(\alpha, \beta)-\langle\alpha, \beta\rangle g\left(V_{\alpha}, V_{\alpha}\right) .
$$

From this, proposition 8 and proposition 9 we get the lemma.

Proposition 10. Put $\mathfrak{v}:=\bigoplus_{\alpha \in \Delta^{+}} \mathfrak{v}_{\alpha}$. Then the following assertions holds.

1) If $g(\mathfrak{t}, \mathfrak{v})=0$ on $(M, g)$, then every $X \in \mathfrak{g}_{1}$ has constant length on $(M, g)$;

2) If the roots of $\mathfrak{g}_{1}$ have one and the same length, and $g\left(\mathfrak{t}, \mathfrak{v}_{\alpha}\right)=0$ for some $\alpha \in \Delta^{+}$, then $g(\mathfrak{t}, \mathfrak{v})=0$ on $(M, g)$.

Proof. For every $X \in \mathfrak{t}$ we have $g\left(X, \mathfrak{v}_{\alpha}\right)=0$ for all $\alpha \in \Delta^{+}$. This obviously implies that $g([X,[X, Y]], X)=0$ on $(M, g)$ for every $Y \in \mathfrak{g}$. Hence, $X$ has constant length on $(M, g)$ by proposition 2 , that proves 1$)$.

Suppose that $g\left(\mathfrak{t}, \mathfrak{v}_{\alpha}\right)=0$ for some $\alpha \in \Delta^{+}$. Let $\eta$ be an inner automorphism of $\mathfrak{g}_{1}$ (and of $\mathfrak{g}$ after the natural extension of the action), such that $\eta(\mathfrak{t})=\mathfrak{t}$. Then $g\left(\mathfrak{t}, \eta\left(\mathfrak{v}_{\alpha}\right)\right)=g\left(\eta(\mathfrak{t}), \eta\left(\mathfrak{v}_{\alpha}\right)\right)=0$ by lemma 5 . Restriction of $\eta$ to $\mathfrak{t}$ is an element $w$ of the Weil group $W$. If $\beta=w(\alpha)$, this mean $g\left(\mathfrak{t}, \mathfrak{v}_{\beta}\right)=0$. Since all $w \in W$ could be obtained by this manner (see section 4 ), then $g\left(\mathfrak{t}, \mathfrak{v}_{w(\alpha)}\right)=0$ for all $w \in W$. Since the Weyl group acts transitively on the set of the roots of a fixed length, then $g(\mathfrak{t}, \mathfrak{v})=0$ for $\mathfrak{g}_{1}$ with the roots of one and the same length.

Remark 3. The list of simple Lie algebras with the roots of one and the same length is the following: $A_{l}=s u(l+1), D_{l}=s o(2 l), e_{6}, e_{7}, e_{8}$.

Now we are ready to finish the proof theorem 2 .

Proposition 11. If the case 2) of proposition 6 holds but 1) does not hold, then the pair $\left(\mathfrak{g}_{1}, \mathfrak{k}\right)$ is one of the following irreducible Hermitian symmetric pair:

1) $(s u(p+q), s u(p) \oplus s u(q) \oplus \mathbb{R}), p \geq q \geq 1$;

2) $(s o(2 n), s u(n) \oplus \mathbb{R}), n \geq 5$;

3) $(s o(p+2), s o(p) \oplus \mathbb{R}), p \geq 5$;

4) $(\operatorname{sp}(n), s u(n) \oplus \mathbb{R}), n \geq 2$.

Proof. There are the following irreducible Hermitian symmetric pair (see [31] or $[32]):(s u(p+q), s u(p) \oplus s u(q) \oplus \mathbb{R}), p \geq q \geq 1 ;(s o(2 n), s u(n) \oplus \mathbb{R}), n \geq 5$; $(s o(p+2), s o(p) \oplus \mathbb{R}), p \geq 5 ;(s p(n), s u(n) \oplus \mathbb{R}), n \geq 2 ;\left(e_{6}, s o(10) \oplus \mathbb{R}\right) ;\left(e_{7}, e_{6} \oplus \mathbb{R}\right)$.

We should exclude the last two pairs. Let us start with the pair $\left(e_{6}, s o(10) \oplus \mathbb{R}\right)$. There are two non-compact roots for $e_{6}: \pi_{1}$ and $\pi_{6}$, but we may consider only first case, because the second is reduced to the first by a suitable automorphism of the Lie algebra $e_{6}$. Hence $Z \in \mathfrak{t}$ is such that $\left\langle Z, \pi_{i}\right\rangle=0$ for $i=2, \ldots, 6$. Up to multiple by a constant we get $Z=\frac{2}{3}\left(e_{8}-e_{7}-e_{6}\right)$, see section 4 .

All root $\alpha=\frac{1}{2}\left(e_{8}-e_{7}-e_{6}+\sum_{i=1}^{5}(-1)^{v_{i}} e_{i}\right)$ with even $\sum_{i=1}^{5} v_{i}$ are in $I_{1}(Z)$. Consider also $\beta=\frac{1}{2}\left(e_{8}-e_{7}-e_{6}+\sum_{i=1}^{5}(-1)^{u_{i}} e_{i}\right)$ with even $\sum_{i=1}^{5} u_{i}, \alpha \neq \beta$. Clear that $\langle\alpha, \beta\rangle=0$ if and only if $\sum_{i=1}^{5}(-1)^{v_{i}+u_{i}}=-3$, that means that $\left(u_{1}, \ldots, u_{5}\right)$ and $\left(v_{1}, \ldots, v_{5}\right)$ are distinct exactly in four coordinates. Put $\left(v_{1}, \ldots, v_{5}\right)=(1,1,1,1,0)$ and let $\left(u_{1}, \ldots, u_{5}\right)$ be such that $u_{5}=1$ and there are exactly one 0 among $u_{i}, 1 \leq i \leq 4$. 
If we consider also $\gamma:=\frac{1}{2}\left(e_{8}-e_{7}-e_{6}+\sum_{i=1}^{5} e_{i}\right)$, then $\langle\alpha, \gamma\rangle=\langle\beta, \gamma\rangle=0$ and $g\left(\alpha, \mathfrak{v}_{\gamma}\right)=g\left(\beta, \mathfrak{v}_{\gamma}\right)=g\left(\alpha-\beta, \mathfrak{v}_{\gamma}\right)=0$ by proposition 9 . The vectors $\alpha-\beta$ are exactly all vectors of the form $e_{5}-e_{i}, 1 \leq i \leq 4$. Moreover, $g\left(\gamma, \mathfrak{v}_{\gamma}\right)=0$ by proposition 9 . Hence, $g\left(\alpha-\gamma, \mathfrak{v}_{\gamma}\right)=0$. Since $\gamma-\alpha=e_{1}+e_{2}+e_{3}+e_{4}$, then $g\left(e_{i}, \mathfrak{v}_{\gamma}\right)=0$ for $1 \leq i \leq 5$. Moreover, $g\left(e_{8}-e_{7}-e_{6}, \mathfrak{v}_{\gamma}\right)=0$, because $e_{8}-e_{7}-e_{6}=2 \gamma-\sum_{i=1}^{5} e_{i}$. Therefore, $g\left(\mathfrak{t}, \mathfrak{v}_{\gamma}\right)=0$ and all $X \in \mathfrak{g}_{1}$ has constant length on $(M, g)$ by proposition 10 . Hence, this case could be excluded.

Now, consider the pair $\left(e_{7}, e_{6} \oplus \mathbb{R}\right)$. There is only one non-compact root for $e_{7}: \pi_{7}$. Hence $Z \in \mathfrak{t}$ is such that $\left\langle Z, \pi_{i}\right\rangle=0$ for $i=1, \ldots, 6$. Up to multiple by a constant we get $Z=\frac{1}{2}\left(e_{8}-e_{7}+2 e_{6}\right)$, see section 4 . It is clear that $e_{8}-e_{7} \in I_{1}(Z)$ and $e_{6} \pm e_{i} \in I_{1}(Z)$ for $1 \leq i \leq 5$. A root $\beta=\frac{1}{2}\left(e_{8}-e_{7}+\sum_{i=1}^{6}(-1)^{v_{i}} e_{i}\right)$ with odd $\sum_{i=1}^{6} v_{i}$ is in $I_{1}(Z)$ if and only if $v_{6}=0$.

Note that $\left\langle e_{8}-e_{7}, e_{6} \pm e_{i}\right\rangle=0$ for $1 \leq i \leq 5$. By proposition 9 , we have $g\left(\alpha, \mathfrak{v}_{\alpha}\right)=0$, where $\alpha=e_{8}-e_{7}$ and $g\left(e_{i}, \mathfrak{v}_{\alpha}\right)=0$ for all $1 \leq i \leq 6$. Therefore, $g\left(\mathfrak{t}, \mathfrak{v}_{\alpha}\right)=0$ on $(M, g)$ and all $X \in \mathfrak{g}_{1}$ has constant length on $(M, g)$ by proposition 10 . Consequently, this case also could be excluded.

Proof of theorem 2. The proof follows immediately from propositions 6, 7, and 11.

\section{Further Study of the Hermitian CASE}

In this section we describe some important properties of Killing vector fields of constant length $Z=Z_{0}+Z_{1}$ for the following Hermitian symmetric pairs:

$$
\begin{aligned}
(s u(p+q), s u(p) \oplus s u(q) \oplus \mathbb{R}), p \geq q \geq 1 ; & (s o(2 n), s u(n) \oplus \mathbb{R}), n \geq 5 ; \\
(s o(p+2), s o(p) \oplus \mathbb{R}), \quad p \geq 5 ; & (s p(n), s u(n) \oplus \mathbb{R}), \quad n \geq 2 .
\end{aligned}
$$

This information could be useful in the forthcoming study of homogeneous Riemannian manifolds with nontrivial Killing vector fields of constant length.

We start with the following question.

Question 1. Let $Z=Z_{0}+Z_{1}$, be a Killing field of constant length on $(M, g)$, where $Z_{0} \in \mathfrak{c}, Z_{1} \in \mathfrak{g}_{1}, Z_{1} \neq 0$ as in theorem 2. Is it true that $Z_{1}$ is also a Killing field of constant length on $(M, g)$ ?

We know that the answer to this question is negative in general (see example 5 in section 1 and proposition 13 below). But the answer is positive for some types of simple Lie algebras $\mathfrak{g}_{1}$. Recall also that the Killing field $Z_{0} \in \mathfrak{c}$ always has constant length on $(M, g)$.

Proposition 12. If, in the above notation, there is an inner automorphism of $\mathfrak{g}_{1}$, such that $\psi\left(Z_{1}\right)=-Z_{1}$, then both Killing fields $Z_{0}-Z_{1}$ and $Z_{1}$ have constant length on $(M, g)$. This is the case when the Weyl group $W$ of $\mathfrak{g}_{1}$ contains the map - Id.

Proof. Indeed, $Z_{0}-Z_{1}=Z_{0}+\psi\left(Z_{1}\right)$ has constant length (by lemma 2) with the same $\mathfrak{k}$ and $\mathfrak{m}$ as $Z=Z_{0}+Z_{1}$ has. Since $g\left(Z_{0} \pm Z_{1}, \mathfrak{m}\right)=0$ by corollary 3 , we get $g\left(Z_{0}, \mathfrak{m}\right)=0$ and $g\left(Z_{1}, \mathfrak{m}\right)=0$. Hence, $Z_{1}$ has constant length by proposition 2 , because $\operatorname{Im}\left(L_{Z}\right)=\operatorname{Im}\left(L_{Z_{1}}\right)=\mathfrak{m}$.

Finally, if $W$ contains the map - Id, then there is an inner automorphism $\eta$ of $\mathfrak{g}$ such that $\eta\left(Z_{1}\right)=-Z_{1}$ by properties of the Weyl group $W$ in section 4 . 
Remark 4. Note that the Weyl group $W$ of a simple Lie algebra $\mathfrak{g}$ does not contain the map $-\mathrm{Id}$, if and only if $\mathfrak{g}$ is one of the following Lie algebras (see e.g. corollary 5.6.3 in [11]): su $(l+1)=A_{l}, l \geq 2$, so $(4 k+2)=D_{2 k+1}, k \geq 1, e_{6}$.

Proposition 13. If, in the above notation, $Z_{1}$ has constant length on $(M, g)$, then $g\left(Z_{0}, \mathfrak{g}_{1}\right)=0$ on $(M, g)$. In particular, if $\mathfrak{g}=\mathfrak{c} \oplus \mathfrak{g}_{1}$ and $M$ is simply connected in addition, then $Z_{0}=0$.

Proof. Since both $Z=Z_{0}+Z_{1}$ and $Z_{1}$ has constant length on $(M, g)$, then $g(Z, \mathfrak{m})=g\left(Z_{1}, \mathfrak{m}\right)=0$, where $\mathfrak{m}$ is the $\langle\cdot, \cdot\rangle$-orthogonal complement to $\mathfrak{k}$ (the centralizer of $Z_{1}$ and $Z$ in $\left.\mathfrak{g}_{1}\right)$, see corollary 3. Hence, $g\left(Z_{0}, \mathfrak{m}\right)=0$ and $0=\mathfrak{m} \cdot g\left(Z_{0}, \mathfrak{m}\right)=$ $g\left(Z_{0},[\mathfrak{m}, \mathfrak{m}]\right)$. Since $\mathfrak{m}+[\mathfrak{m}, \mathfrak{m}]$ is an ideal in $\mathfrak{g}_{1}$ by lemma 6 , then $\mathfrak{m}+[\mathfrak{m}, \mathfrak{m}]=\mathfrak{g}_{1}$ and consequently, $g\left(Z_{0}, \mathfrak{g}_{1}\right)=0$. The second assertion follows from the fact that $\mathfrak{g}_{1}$ acts transitively on simply connected $M$ (see e.g. proposition 9 on p. 94 in [24]).

Now, we are going to discuss four Hermitian pairs in theorem 2 in more details.

1. The pair $(s u(p+q), s u(p) \oplus s u(q) \oplus \mathbb{R}), p \geq q \geq 1$. For the Lie algebra $s u(p+q)$, all simple roots $\pi_{i}, 1 \leq i \leq p+q-1$, are non-compact, see section 4 . Let us fix $i=p$. Then $Z_{1}$ is $\langle\cdot, \cdot\rangle$-orthogonal to all $\pi_{j}=e_{j}-e_{j+1}, j \neq p$. Up to multiplication by a constant, we have

$$
Z_{1}=\frac{1}{p+q}\left(q\left(e_{1}+e_{2}+\cdots+e_{p}\right)-p\left(e_{p+1}+\cdots+e_{p+q}\right)\right) .
$$

A root $\alpha=e_{i}-e_{j}$ is in $I_{1}(Z) \subset \Delta^{+}$if and only if $i \leq p$ and $j \geq p+1$ (in such a case $\langle Z, \alpha\rangle=1)$.

Now, suppose that $q \geq 2$ and consider pairwise distinct indices $i, j, k, l$. Using properties of the Weyl group $W$ of $s u(p+q)$ (which is the permutation group of the vectors $\left.e_{i}, 1 \leq i \leq p+q\right)$, we can choose a Killing fields of constant length of the type $Z_{0}+w\left(Z_{1}\right), w \in W$, (see lemma 10) in such a way, that $\alpha, \beta \in I_{1}\left(Z_{0}+w\left(Z_{1}\right)\right.$ ), where $\alpha=\left|e_{i}-e_{j}\right|$ and $\beta=\left|e_{k}-e_{l}\right|$. Since $\left[\mathfrak{v}_{\alpha}, \mathfrak{v}_{\beta}\right]=0$ and $\langle\alpha, \beta\rangle=0$, then we get

$$
g\left(\mathfrak{v}_{\alpha}, \mathfrak{v}_{\beta}\right)=g\left(\mathfrak{v}_{\alpha}, \beta\right)=g\left(\alpha, \mathfrak{v}_{\beta}\right)=g(\alpha, \beta)=0
$$

on $(M, g)$ by lemmas 11,12 and proposition 9 . In particular, there are $q$ roots $\alpha_{i}$, $1 \leq i \leq q$ such that $g\left(\alpha_{i}, \alpha_{j}\right)=0$ on $(M, g)$ for $i \neq j$.

Note also that $g(\alpha, \alpha)=\langle\alpha, \alpha\rangle g(\alpha, Z)=2 g(\alpha, Z)$ by proposition 8 for any $\alpha=$ $e_{i}-e_{j} \in I_{1}(Z)$. Now, we suppose that $Z_{0}=0$. Then, using the explicit form of $Z=Z_{1}(16)$ and (17) we get

$$
\begin{array}{r}
\frac{p+q}{2} g\left(e_{i}-e_{j}, e_{i}-e_{j}\right)=(p+q) g\left(Z, e_{i}-e_{j}\right) \\
=g\left(\left(e_{i}-e_{j}\right)+\sum_{k \leq p, k \neq i}\left(e_{k}-e_{j}\right)+\sum_{l>p, l \neq j}\left(e_{i}-e_{l}\right), e_{i}-e_{j}\right)
\end{array}
$$

Let us fix $r \leq p$ and $s>p$ such that $r \neq i$ and $s \neq j$, then using (17) again, we get

$$
\begin{array}{r}
\frac{p+q}{2} g\left(e_{i}-e_{j}, e_{i}-e_{j}\right)=(p+q) g\left(Z, e_{i}-e_{j}\right)=g\left(e_{i}-e_{j}, e_{i}-e_{j}\right) \\
+g\left(\sum_{k \leq p, k \neq i}\left(\left(e_{k}-e_{r}\right)+\left(e_{r}-e_{j}\right)\right)+\sum_{l>p, l \neq j}\left(\left(e_{i}-e_{s}\right)+\left(e_{s}-e_{l}\right)\right), e_{i}-e_{j}\right) \\
=g\left(e_{i}-e_{j}, e_{i}-e_{j}\right)+(p-1) g\left(e_{r}-e_{j}, e_{i}-e_{j}\right)+(q-1) g\left(e_{i}-e_{s}, e_{i}-e_{j}\right),
\end{array}
$$


that is equivalent to

$$
(p+q-2) g\left(e_{i}-e_{j}, e_{i}-e_{j}\right)=2(p-1) g\left(e_{r}-e_{j}, e_{i}-e_{j}\right)+2(q-1) g\left(e_{i}-e_{s}, e_{i}-e_{j}\right) .
$$

Using the Weyl group as above, we see that (18) is fulfilled for every distinct $i, j, r, s$. Interchanging $i$ and $j$, as well as $r$ and $s$, in (18), we get

$$
(p+q-2) g\left(e_{i}-e_{j}, e_{i}-e_{j}\right)=2(q-1) g\left(e_{r}-e_{j}, e_{i}-e_{j}\right)+2(p-1) g\left(e_{i}-e_{s}, e_{i}-e_{j}\right) .
$$

Subtracting (18) from the this equality, we get $(p-q) g\left(e_{r}+e_{s}-e_{i}-e_{j}, e_{i}-e_{j}\right)=0$, that implies $g\left(e_{r}-e_{j}, e_{i}-e_{j}\right)=g\left(e_{i}-e_{s}, e_{i}-e_{j}\right)$ for $p \neq q$. From this and (18) we get

$$
(p+q-2) g\left(e_{i}-e_{j}, e_{i}-e_{j}\right)=2(p+q-2) g\left(e_{i}-e_{s}, e_{i}-e_{j}\right),
$$

that implies $g\left(e_{i}-e_{j}, e_{i}-e_{j}-2\left(e_{i}-e_{s}\right)\right)=0$. Put $\alpha=e_{i}-e_{j}$, then we get $g\left(\mathfrak{v}_{\alpha}, \alpha\right)=0$ and $g\left(\mathfrak{v}_{\alpha}, e_{i}-e_{s}\right)=0$ for any $s \neq i$ by proposition 9 . Therefore, $g\left(\mathfrak{v}_{\alpha}, \mathfrak{t}\right)=0$ and every $X \in \mathfrak{g}_{1}=s u(p+q)$ has constant length on $(M, g)$ by proposition 10 . Then we get

Proposition 14. If $Z=Z_{1}$ (i.e. $\left.Z_{0}=0\right)$ for the pair $(s u(p+q)$, su $(p) \oplus s u(q) \oplus \mathbb{R})$ and $p \neq q \geq 2$, then every $X \in \mathfrak{g}_{1}=\operatorname{su}(p+q)$ has constant length on $(M, g)$.

Note also that for $p=q$ there is $w \in W$ such that $w\left(Z_{1}\right)=-Z_{1}$, hence, $Z_{1}$ is a Killing field of constant length on $(M, g)$ by proposition 12 .

2. The pair $(s o(2 n), s u(n) \oplus \mathbb{R}), n \geq 5$. For the Lie algebra $s o(2 n)$, the are three non-compact roots, $\pi_{1}$ and $\pi_{n-1}$, and $\pi_{n}$, see section 4 . For this pair we should choose $\pi_{n}\left(\pi_{n-1}\right.$ lead to the same result due to a suitable automorphism of $s o(2 n)$, and $\pi_{1}$ corresponds to another pair). Then $Z_{1}$ is $\langle\cdot, \cdot\rangle$-orthogonal to all $\pi_{i}=e_{i}-e_{i+1}, i<n$. Up to multiplication by a constant, we have

$$
Z_{1}=\frac{1}{2}\left(e_{1}+e_{2}+\cdots+e_{n-1}+e_{n}\right)
$$

Clear that $I_{1}(Z) \subset \Delta^{+}$consists of the root $\alpha=e_{i}+e_{j}, 1 \leq i<j \leq n$, and $\langle Z, \alpha\rangle=1$ for such $\alpha$.

Let us choose distinct indices $i, j, k, l$ and put $\alpha=e_{i}+e_{j}, \beta=e_{k}+e_{l}$. Since $\left[\mathfrak{v}_{\alpha}, \mathfrak{v}_{\beta}\right]=0$ and $\langle\alpha, \beta\rangle=0$, then we get

$$
g\left(\mathfrak{v}_{\alpha}, \mathfrak{v}_{\beta}\right)=g\left(\mathfrak{v}_{\alpha}, \beta\right)=g\left(\alpha, \mathfrak{v}_{\beta}\right)=g(\alpha, \beta)=g\left(e_{i}+e_{j}, e_{k}+e_{l}\right)=0
$$

on $(M, g)$ by lemmas 11,12 and proposition 9 . The Weyl group $W$ permutes $e_{i}, 1 \leq$ $i \leq n$, and changes the signs of even numbers of the basic vectors. Using $Z_{0}+w(W)$ for a suitable $w \in W$ instead of $Z=Z_{0}+Z_{1}$, we easily get that $g\left(e_{i}+e_{j}, e_{k}-e_{l}\right)=0$ too (here we have used that $n \geq 5$ ). Therefore, $g\left(e_{i}+e_{j}, e_{k}\right)=0$. Using the Weyl group one more time, we get $g\left(e_{i}, e_{k}\right)=0$ on $(M, g)$ for any $i \neq k$.

Proposition 15. If a Killing field $Z=Z_{0}+Z_{1}$ has constant length on $(M, g)$ for the pair $(s o(2 n), s u(n) \oplus \mathbb{R})$, then there exist $n$ pairwise commuting $U_{i} \in s o(2 n)$, $1 \leq i \leq n$, such that $\left\langle U_{i}, U_{j}\right\rangle=\delta_{i j}, Z_{1}=c \cdot \sum_{i=1}^{n} U_{i}$ for some positive $c \in \mathbb{R}$ and $g\left(U_{i}, U_{j}\right)=0$ for $i \neq j$ on $(M, g)$.

Note that $\delta_{i j}$ above means the Kronecker delta, i.e. $\delta_{i j}=1$ for $i=j$ and $\delta_{i j}=$ 0 for $i \neq j$. It should be noted also that for even $n$, the Weyl group contained - Id, Therefore, $Z_{1}$ is also Killing field of constant length on $(M, g)$ in this case by proposition 12 . 
3. The pair $(s o(p+2), s o(p) \oplus \mathbb{R}), p \geq 5$. In this case we should distinguish the subcase $p=2 l-2$ for some integer $l \geq 4$ and the subcase $p=2 l-1$ for some integer $l \geq 3$.

If $s o(p+2)=s o(2 l)$, we should consider non-compact root $\pi_{1}=e_{1}-e_{2}$, see section 4 . Then $Z_{1}$ is $\langle\cdot, \cdot\rangle$-orthogonal to all $\pi_{i}, 2 \leq i \leq n$. Up to multiplication by a constant, we have

$$
Z_{1}=e_{1}
$$

Clear that $I_{1}(Z) \subset \Delta^{+}$consists of the root $\alpha=e_{1} \pm e_{i}, 2 \leq i \leq n$, and $\langle Z, \alpha\rangle=1$ for such $\alpha$.

If $s o(p+2)=s o(2 l+1)$, a unique non-compact root in $s o(2 l+1)$ is also $\pi_{1}=e_{1}-e_{2}$ and we have $Z$ as in (21). Note, however, that in this subcase $Z_{1}$ is also the root. $I_{1}(Z) \subset \Delta^{+}$consists of the roots $\alpha=e_{1} \pm e_{i}, 2 \leq i \leq n$, and $e_{1}$.

The next arguments work both $s o(p+2)=s o(2 l)$ and $s o(p+2)=s o(2 l+1)$. Let $F_{i, j}$ be a $(p+2) \times(p+2)$-matrix $\left(a_{t, s}\right)$ with all zero entries excepting $a_{i, j}=-1$ and $a_{j, i}=1$. We will identify $F_{2 i-1,2 i}$ with $e_{i}$ in $s o(p+2)$, see e.g. chapter 8 in [4]. Hence $F_{1,2}=e_{1}=Z_{1}$. If we take $a=\operatorname{diag}\left(\left(\begin{array}{ll}0 & 1 \\ 1 & 0\end{array}\right), 1, \ldots, 1\right) \in S O(p+2)$, then $\operatorname{Ad}(a)\left(F_{1,2}\right)=-F_{1,2}$. Therefore, $Z_{1}=F_{1,2}$ is also constant length on $(M, g)$ by proposition 12. In what follows, we assume that $Z=Z_{1}=F_{1,2}$.

Now, for all $a \in S O(p+2)$ and all $c \in \mathbb{R}$, the vector $\operatorname{Ad}(a)\left(c F_{1,2}\right)$ has constant length on $(M, g)$ by lemma 5 . From this observation we get that all $X \in \mathfrak{f}$ have constant length on $(M, g)$, where $\mathfrak{f}$ is the subalgebra of $s o(p+2)$, spanned by the Killing vector fields $F_{1,2}, F_{2,3}$, and $F_{1,3}$.

For every $X, Y \in \mathfrak{f}$ we have $2 g(X, Y)=g(X+Y, X+Y)-g(X, X)-g(Y, Y)=$ const, because all $X \in \mathfrak{f}$ have constant length. Hence, $2 \nabla_{X} Y=[X, Y] \in \mathfrak{f}$ by lemma 3. This means that the distribution $\mathfrak{f}$ is autoparallel on $(M, g)$.

Moreover, $R(X, Y) Y=-\nabla_{Y} \nabla_{Y} X=-\frac{1}{4}[Y,[Y, X]]$ by lemma 4 . But it is easy to see that $[Y,[Y, X]]=-X$ for any $X, Y \in \mathfrak{f}$ such that $\langle X, X\rangle=\langle Y, Y\rangle=\left\langle F_{1,2}, F_{1,2}\right\rangle$ and $\langle X, Y\rangle=0$ ( $\mathfrak{f}$ is isomorphic to $s o(3))$. Therefore, $R(X, Y) Y=\frac{1}{4} X$. Moreover, $g(X, Y)=0$ by proposition 2 and $g(Y, Y)=g\left(F_{1,2}, F_{1,2}\right)$ by lemma 5 . Hence, the sectional curvature of the plane spanned by $X$ an $Y$ (at every point $x \in M$ ) is equal

$$
\frac{g(R(X, Y) Y, X)}{g(X, X) g(Y, Y)-g(X, Y)^{2}}=\frac{1}{4 g(Y, Y)}=\frac{1}{4 g\left(F_{1,2}, F_{1,2}\right)}=\text { const . }
$$

It is clear, that the subalgebra $\psi(\mathfrak{f})$ in $s o(p+2)$, where $\psi$ is any inner automorphism of $s o(p+2)$, also consist of Killing vector fields of constant length. In particular, any $F_{i, j} \in \operatorname{so}(p+2), i \neq j$, has constant length on $(M, g)$. From this we get

$$
0=F_{k, j} \cdot g\left(F_{i, k}, F_{i, k}\right)=2 g\left(\left[F_{k, j}, F_{i, k}\right], F_{i, k}\right)=2 g\left(F_{i, j}, F_{i, k}\right), \quad i \neq k .
$$

therefore, the linear subspace $P_{i}:=\operatorname{Lin}\left(F_{i, j} \mid j \neq i\right)$ in $s o(p+2)$ consists of Killing vector fields of constant length. Therefore, we get

Proposition 16. If a Killing vector field $Z=Z_{0}+Z_{1}$ has constant length on $(M, g)$ for the pair $(s o(p+2)$, so $(p) \oplus \mathbb{R})$, then the following assertions hold:

1) The Killing field $Z_{1}$ has constant length too;

2) There is a 3-dimensional Lie subalgebra $\mathfrak{f}$ in $s o(p+2)$, that consists of Killing fields of constant length and such that all orbits of the subgroup $\exp (\mathfrak{f}) \subset \exp (\mathfrak{g})=G$ in $(M, g)$ are totally geodesic and have (one and the same) constant positive curvature; 
3) There are $(p+1)$-dimensional linear subspaces in so $(p+2)$, that consist of Killing vector fields of constant length.

Note that linear subspaces, that consist of Killing vector fields of constant length are called Clifford-Killing spaces in [8], where Clifford-Killing spaces for Euclidean spheres were studied in particular. The authors of [34] use the term Clifford-Killing vector fields for Killing vector fields of constant length.

4. The pair $(s p(n), s u(n) \oplus \mathbb{R}), n \geq 2$. A unique non-compact root in $s p(n)$ is $\pi_{n}=2 e_{n}$. Therefore, $Z_{1}$ is $\langle\cdot, \cdot\rangle$-orthogonal to all $\pi_{i}=e_{i}-e_{i+1}, i<n$. Up to multiplication by a constant, we have

$$
Z_{1}=\frac{1}{2}\left(e_{1}+e_{2}+\cdots+e_{n-1}+e_{n}\right)
$$

Clear that $I_{1}(Z) \subset \Delta^{+}$consists of the roots $2 e_{i}, 1 \leq i \leq n$, and $e_{i}+e_{j}, 1 \leq i<j \leq n$. Let us choose distinct indices $i, j$ and put $\alpha=2 e_{i}, \beta=2 e_{j}$. Since $\left[\mathfrak{v}_{\alpha}, \mathfrak{v}_{\beta}\right]=0$ and $\langle\alpha, \beta\rangle=0$, then we get

$$
g\left(\mathfrak{v}_{\alpha}, \mathfrak{v}_{\beta}\right)=g\left(\mathfrak{v}_{\alpha}, \beta\right)=g\left(\alpha, \mathfrak{v}_{\beta}\right)=g(\alpha, \beta)=4 g\left(e_{i}, e_{j}\right)=0
$$

on $(M, g)$ by lemmas 11,12 and proposition 9 .

Since the Weyl group $W$ for $\operatorname{sp}(n)$ contains the map - Id, then the Killing fields $Z_{1}$ also has constant length on $(M, g)$ by proposition 12 .

Proposition 17. If a Killing field $Z=Z_{0}+Z_{1}$ has constant length on $(M, g)$ for the pair $(\operatorname{sp}(n), s u(n) \oplus \mathbb{R})$, then the Killing field $Z_{1}$ also has constant length on $(M, g)$ and there exist $n$ pairwise commuting $U_{i} \in \operatorname{sp}(n), 1 \leq i \leq n$, such that $\left\langle U_{i}, U_{j}\right\rangle=\delta_{i j}$, $Z_{1}=c \cdot \sum_{i=1}^{n} U_{i}$ for some positive $c \in \mathbb{R}$ and $g\left(U_{i}, U_{j}\right)=0$ for $i \neq j$ on $(M, g)$.

\section{UNSOLVED QUESTIONS}

Results of this paper can be used for the classification of homogeneous Riemannian manifold with Killing vector fields of some special kinds. We state some unsolved questions and problems in this direction.

Problem 1. Classify homogeneous Riemanninan spaces $(G / H, g)$ with nontrivial Killing vector fields of constant length, where $G$ is simple.

Recall, that normal homogeneous Riemanninan spaces $(G / H, g)$ with Killing fields of constant length, where $G$ is simple, are classified in [34]. On the other hand, the set of $G$-invariant metrics on a space $G / H$ could have any dimension even for simple $G$.

Question 2. Let $(M, g)$ be a Riemannian homogeneous manifold, $G$ be its full connected isometry group, and $Z \in \mathfrak{g}$ be a Killing vector field of constant length on $(M, g)$. Does $\mathfrak{k}$, the centralizer of $Z$ in $\mathfrak{g}$, acts transitively on $M$ ?

Note, that for symmetric spaces $(M=G / H, g)$ we have an affirmative answer to this question (see lemma 3 in [6]). Of course, question 2 is interesting even under an additional assumption that the group $G$ is simple.

There is a natural generalization of normal homogeneous spaces. Recall, that a Riemannian manifold $(M=G / H, g)$, where $H$ is a compact subgroup of a Lie group $G$ and $g$ is a $G$-invariant Riemannian metric, is called a geodesic orbit space if any 
geodesic $\gamma$ of $M$ is an orbit of 1-parameter subgroup of the group $G$, detailed information on this class of homogeneous Riemannian manifolds one can find e.g. in $[1,2,9,20,27]$. It is known that all normal homogeneous space are geodesic orbit. The following problem is natural.

Problem 2. Classify geodesic orbit Riemannian space with nontrivial Killing vector fields of constant length.

Recall the following result of [23]: If $(M=G / H, g)$ is a geodesic orbit Riemannian space, $\mathfrak{g}$ is its Lie algebra of Killing fields, and $\mathfrak{a}$ is an abelian ideal in $\mathfrak{g}$, then every $X \in \mathfrak{a}$ has constant length on $(M, g)$. Hence it suffices to study only homogeneous spaces $G / H$ with semisimple $G$ in problem 2 .

Note that the examples from section 1 cover all possibilities in theorem 2, excepting the case 3), where we have examples only for specific values of $p$.

Question 3. Is there a Riemannian homogeneous space $(M=G / H, g)$ with a Killing vector field of constant length $Z \in \mathfrak{g}$, corresponded to the case 3) in theorem 2, i.e. $\left(\mathfrak{g}_{1}, \mathfrak{k}\right)=(s o(p+2), s o(p) \oplus \mathbb{R})$, for $p=6$ and $p \geq 8$ ?

For $p=5$ and $p=7$ see examples 6 and 7 . Note that proposition 16 gives serious restrictions on a Riemannian manifold $(M=G / H, g)$ as in question 3.

\section{REFERENCES}

[1] Alekseevsky D.V., Arvanitoyeorgos A. Riemannian flag manifolds with homogeneous geodesics, Trans. Amer. Math. Soc., 359 (2007), 3769-3789 (2007).

[2] Alekseevsky D.V., Nikonorov Yu.G. Compact Riemannian manifolds with homogeneous geodesics. SIGMA Symmetry Integrability Geom. Methods Appl., 5, 093, 16 pages (2009). Online access: http://www.emis.de/journals/SIGMA/2009/093/

[3] Belgun F., Moroianu A., Semmelmann U. Symmetries of contact metric manifolds. Geom. Dedicata, 101, 203-216 (2003).

[4] Besse A.L. Einstein manifolds, Springer, Berlin (1987).

[5] Berestovskii V.N., Nikonorov Yu.G. Killing vector fields of constant length on Riemannian manifolds. Siber. Math. J., 49(3), 395-407 (2008).

[6] Berestovskii V.N., Nikonorov Yu.G. Killing vector fields of constant length on locally symmetric Riemannian manifolds. Transform. Groups, 13(1), 25-45 (2008).

[7] Berestovskii V.N., Nikonorov Yu.G. Regular and quasiregular isometric flows on Riemannian manifolds. Matem. tr., 10(2), 3-18 (2007) (Russian); English translation in: Siberian Adv. Math., 18(3), 153-162 (2008).

[8] Berestovskii V.N., Nikonorov Yu.G. Clifford-Wolf homogeneous Riemannian manifolds. J. Differ. Geom., 82(3), 467-500 (2009).

[9] Berestovskii V.N., Nikonorov Yu.G. Riemannian manifolds and homogeneous geodesics, South Mathematical Institute of VSC RAS, Vladikavkaz, 2012, 412 p. (Russian). Online access: http://rucont.ru/efd/230575

[10] Berestovskii V.N., Nikonorov Yu.G. Generalized normal homogeneous Riemannian metrics on spheres and projective spaces. Ann. Glob. Anal. Geom., 45(3), 167-196 (2014).

[11] Bourbaki N. Éléments de mathématique. Fasc. XXXIV. Groupes et algèbres de Lie. Chapitres IV, V et VI: Groupes de Coxeter et systèmes de Tits. Groupes engendrés par des réflexions. Systèmes de racines. Paris: Hermann \& Cie (1968).

[12] Bourbaki, N. Éléments de mathématique. Groupes et algèbres de Lie, Chapitre 9: Groupes de Lie réels compacts. Paris etc.: Masson (1982).

[13] Deng S., Xu M. Clifford-Wolf homogeneous Randers spaces. J. Lie Theory 23(3), 837-845 (2013). 
[14] Deng S., Xu M. Clifford-Wolf translations of homogeneous Randers spheres. Israel J. Math. 199(2), 507-525 (2014).

[15] Deng $S ., \mathrm{Xu} M$. Clifford-Wolf translations of left invariant Randers metrics on compact Lie groups. Q. J. Math. 65(1), 133-148 (2014).

[16] Freudenthal H. Clifford-Wolf-Isometrien symmetrischer Raume, Math. Ann. 150, 136-149 (1963)(German).

[17] Helgason S. Differential geometry, Lie groups, and symmetric spaces. New York-San FranciscoLondon: Academic Press (1978).

[18] Kobayashi S., Nomizu K. Foundations of differential geometry. Vol. I, A Wiley-Interscience Publication, New York (1963); Vol. II, A Wiley-Interscience Publication, New York (1969).

[19] Kostant B. On holonomy and homogeneous spaces. Nagoya Math. J., 12, 31-54 (1957).

[20] Kowalski O., Vanhecke L. Riemannian manifolds with homogeneous geodesics. Boll. Un. Mat. Ital. B (7), 5(1), 189-246 (1991).

[21] Ledger A.J., Obata M. Affine and Riemannian s-manifolds. J. Differential Geom. 2(4), 451-459 (1968).

[22] Nikonorov Yu.G. Invariant Einstein metrics on the Ledger - Obata spaces // Algebra i analiz. 14(3), 169-185 (2002) (Russian); English translation in: St. Petersburg Math. J. 14(3), 487-497 (2003).

[23] Nikonorov Yu.G. Geodesic orbit manifolds and Killing fields of constant length. Hiroshima Math. J., 43(1), 129-137 (2013).

[24] Onishchik A.L. Topology of transitive transformation groups, Johann Ambrosius Barth, Leipzig, Berlin, Heidelberg (1994).

[25] Ozols V. Clifford translations of symmetric spaces. Proc. Amer. Math. Soc. 44, 169-175 (1974).

[26] Samelson H. Notes on Lie algebras. 2nd. ed. Springer, New York etc. (1990).

[27] Tamaru H. Riemannin g. o. spaces fibered over irreducible symmetric spaces. Osaka J. Math., 15, 55-67 (1998).

[28] Wang M., Ziller W. Existence and Non-existence of Homogeneous Einstein Metrics, Invent. Math. 84, 177-194 (1986).

[29] Wolf J.A. Vincent's conjecture on Clifford translations of the sphere. Comment. Math. Helv. 36, 33-41 (1961).

[30] Wolf J.A. Locally symmetric homogeneous spaces, Comment. Math. Helv. 37, 65-101 $(1962 / 1963)$.

[31] Wolf J.A. On the classification of Hermitian symmetric spaces. J. Math. Mech. 13, 489-495 (1964).

[32] Wolf J.A. Spaces of constant curvature. Sixth edition. AMS Chelsea Publishing, Providence, RI, (2011).

[33] Wolf J.A., Podestà F., Xu M. Toward a classification of Killing vector fields of constant length on pseudo-Riemannian normal homogeneous spaces. Preprint, arXiv 1503.08267.

[34] Xu M., Wolf J.A. Killing vector fields of constant length on Riemannian normal homogeneous spaces. Preprint, arXiv 1412.3177.

Yu. G. NIKONOROV

South Mathematical Institute of Vladikavkaz Scientific Centre

of the Russian Academy of Sciences, Vladikavkaz, Markus st. 22, 362027, RUSSIA

E-mail address: nikonorov2006@mail.ru 